\title{
Stability and Hopf Bifurcation in a Prey-Predator System with Disease in the Prey and Two Delays
}

\begin{abstract}
Juan Liu
Department of Mathematics and Physics, Bengbu College, Bengbu 233030, China

Correspondence should be addressed to Juan Liu; liujuan7216@163.com

Received 23 December 2013; Accepted 19 February 2014; Published 6 April 2014

Academic Editor: Wenchang Sun

Copyright (C) 2014 Juan Liu. This is an open access article distributed under the Creative Commons Attribution License, which permits unrestricted use, distribution, and reproduction in any medium, provided the original work is properly cited.

This paper is concerned with a prey-predator system with disease in the prey and two delays. Local stability of the positive equilibrium of the system and existence of local Hopf bifurcation are investigated by choosing different combinations of the two delays as bifurcation parameters. For further investigation, the direction and the stability of the Hopf bifurcation are determined by using the normal form method and center manifold theorem. Finally, some numerical simulations are given to support the theoretical analysis.
\end{abstract}

\section{Introduction}

The effect of disease in ecological system is an important issue from mathematical as well as ecological point of view. Therefore, the dynamics of epidemiological models have been investigated by many authors in recent years [1-6]. In [6], Jana and Kar proposed and investigated the following predatorprey system with disease in the prey:

$$
\begin{gathered}
\frac{d S(t)}{d t}=r S\left(1-\frac{S}{K}\right)-\frac{\alpha S I(t-\tau)}{a+S}-\frac{\beta S P}{b+S}, \\
\frac{d I(t)}{d t}=\frac{\alpha S I(t-\tau)}{a+S}-\gamma I, \\
\frac{d P(t)}{d t}=\frac{m \beta S P}{b+S}-\delta P^{2}-\varepsilon P,
\end{gathered}
$$

where $S(t), I(t)$ denote the population densities of the susceptible prey and the infected prey at time $t$, respectively. $P(t)$ denotes the population of the predator at time $t$. The susceptible prey grows logistically with the intrinsic growth rate $r$ and the carrying capacity $K$. The conversion from the susceptible prey to the infected prey is governed by the response function $\alpha I /(a+S)$. The consumption of the susceptible prey by the predator is governed by the response function $\beta P /(b+S) . \gamma$ is the removal rate of the infected prey biomass. $\delta$ is the intraspecific competition coefficient of the predator. $\varepsilon$ is the removal rate of the predator due to natural death or harvesting. And the constant $\tau(\tau \geq 0)$ is the time delay due to susceptible prey which becomes the infected prey. The predator-prey system with single delay has been investigated by many researchers [7-11]. Jana and Kar [6] studied the boundedness of the solutions and stability of the positive equilibrium of system (1). Existence and properties of the Hopf bifurcation were also investigated.

In recent years, there are also some papers on the bifurcations of a prey-predator system with two or multiple delays [12-16]. As is known to all, the consumption of the susceptible prey by the predator throughout its past history governs the present birth rate of the predator. Therefore, it is reasonable to incorporate time delay due to the gestation of the predator into system (1). Based on this consideration, we consider the following system with two delays in this paper:

$$
\begin{gathered}
\frac{d S(t)}{d t}=r S\left(1-\frac{S}{K}\right)-\frac{\alpha S I\left(t-\tau_{1}\right)}{a+S}-\frac{\beta S P}{b+S}, \\
\frac{d I(t)}{d t}=\frac{\alpha S I\left(t-\tau_{1}\right)}{a+S}-\gamma I, \\
\frac{d P(t)}{d t}=P\left[\frac{m \beta S\left(t-\tau_{2}\right)}{b+S\left(t-\tau_{2}\right)}-\delta P-\varepsilon\right],
\end{gathered}
$$


where $\tau_{1} \geq 0$ is the time delay due to susceptible prey which becomes the infected prey and $\tau_{2} \geq 0$ is the time delay due to the gestation of the predator.

This paper is organized as follows. In Section 2, we investigate local stability of the positive equilibrium and existence of local Hopf bifurcation of system (2) with respect to both delays. In Section 3, by using the normal form method and center manifold theorem, the properties of the Hopf bifurcation such as direction and stability are determined. Some numerical simulations are given for the support of the analytical findings in Section 4.

\section{Local Stability and Hopf Bifurcation}

According to the analysis in [6], system (2) has a unique positive equilibrium $E^{*}\left(S^{*}, I^{*}, P^{*}\right)$ if $\alpha>\gamma, a m \beta \gamma /(b+(a-$ b) $\gamma)>\varepsilon$ and $\left(1-S^{*} / K\right)>\beta P^{*} /\left(b+S^{*}\right)$, where $S^{*}=$ $a \gamma /(\alpha-\gamma), I^{*}=\left(r\left(1-S^{*} / K\right)-\beta P^{*} /\left(b+S^{*}\right)\right)\left(a+S^{*}\right) / \alpha$, $P^{*}=\left(m \beta S^{*}-\varepsilon\left(b+S^{*}\right)\right) /\left(\delta\left(b+S^{*}\right)\right)$.

Let $\bar{S}=S-S^{*}, \bar{I}=I-I^{*}, \bar{P}=P-P^{*}$. Dropping the bars for convenience, system (2) becomes the following form:

$$
\begin{gathered}
\frac{d S(t)}{d t}=a_{1} S(t)+a_{2} P(t)+b_{1} I\left(t-\tau_{1}\right)+F_{1}, \\
\frac{d I(t)}{d t}=a_{3} S(t)+a_{4} I(t)+b_{2} I\left(t-\tau_{1}\right)+F_{2}, \\
\frac{d P(t)}{d t}=a_{5} P(t)+c_{1} S\left(t-\tau_{2}\right)+F_{3},
\end{gathered}
$$

where

$$
\begin{aligned}
a_{1}= & -\frac{r S^{*}}{K}+\frac{\alpha S^{*} I^{*}}{\left(a+S^{*}\right)^{2}}+\frac{\beta S^{*} P^{*}}{\left(b+S^{*}\right)^{2}}, \quad a_{2}=-\frac{\beta S^{*}}{b+S^{*}}, \\
a_{3}= & \frac{a \alpha I^{*}}{\left(a+S^{*}\right)^{2}}, \quad a_{4}=-\gamma, \quad a_{5}=-\delta P^{*}, \\
b_{1}= & -\frac{\alpha S^{*}}{a+S^{*}}, \quad b_{2}=\frac{\alpha S^{*}}{a+S^{*}}, \quad c_{1}=\frac{m b \beta P^{*}}{\left(b+S^{*}\right)^{2}}, \\
F_{1}= & a_{21} S^{2}(t)+a_{22} S(t) I\left(t-\tau_{1}\right)+a_{23} S(t) P(t)+a_{31} S^{3}(t) \\
& +s_{32} S^{2}(t) I\left(t-\tau_{1}\right)+a_{33} S^{2}(t) P(t)+\cdots, \\
F_{2}= & b_{21} S^{2}(t)+b_{22} S(t) I\left(t-\tau_{1}\right)+b_{31} S^{3}(t) \\
& +b_{32} S^{2}(t) I\left(t-\tau_{1}\right)+\cdots, \\
F_{3}= & c_{21} P^{2}(t)+c_{22} P(t) S\left(t-\tau_{2}\right)+c_{23} S^{2}\left(t-\tau_{2}\right) \\
& +c_{31} P(t) S^{2}\left(t-\tau_{2}\right)+c_{32} S^{3}\left(t-\tau_{2}\right)+\cdots,
\end{aligned}
$$

with

$$
\begin{aligned}
& a_{21}=-\frac{r}{K}+\frac{a \alpha I^{*}}{\left(a+S^{*}\right)^{3}}+\frac{b \beta P^{*}}{\left(b+S^{*}\right)^{3}}, \quad a_{22}=-\frac{a \alpha}{\left(a+S^{*}\right)^{3}}, \\
& a_{23}=-\frac{b \beta}{\left(b+S^{*}\right)^{2}}, \quad a_{31}=-\frac{a \alpha I^{*}}{\left(a+S^{*}\right)^{4}}-\frac{b \beta P^{*}}{\left(b+S^{*}\right)^{4}},
\end{aligned}
$$

$$
\begin{aligned}
& a_{32}=\frac{a \alpha}{\left(a+S^{*}\right)^{3}}, \quad a_{33}=\frac{b \beta}{\left(b+S^{*}\right)^{3}}, \\
& b_{21}=-\frac{a \alpha I^{*}}{\left(a+S^{*}\right)^{3}}, \quad b_{22}=\frac{a \alpha}{\left(a+S^{*}\right)^{2}}, \\
& b_{31}=-\frac{a \alpha I^{*}}{\left(a+S^{*}\right)^{4}}, \quad b_{32}=-\frac{a \alpha}{\left(a+S^{*}\right)^{4}}, \\
& c_{21}=-\delta, \quad c_{22}=\frac{m b \beta}{\left(b+S^{*}\right)^{2}}, \quad c_{23}=-\frac{m b \beta P^{*}}{\left(b+S^{*}\right)^{3}}, \\
& c_{31}=-\frac{m b \beta}{\left(b+S^{*}\right)^{3}}, \quad c_{32}=\frac{m b \beta P^{*}}{\left(b+S^{*}\right)^{4}} .
\end{aligned}
$$

The linearized system of (3) is

$$
\begin{gathered}
\frac{d S(t)}{d t}=a_{1} S(t)+a_{2} P(t)+b_{1} I\left(t-\tau_{1}\right), \\
\frac{d I(t)}{d t}=a_{3} S(t)+a_{4} I(t)+b_{2} I\left(t-\tau_{1}\right), \\
\frac{d P(t)}{d t}=a_{5} P(t)+c_{1} S\left(t-\tau_{2}\right) .
\end{gathered}
$$

Thus, we can get that the characteristic equation of system (7) is

$$
\begin{aligned}
\lambda^{3} & +m_{2} \lambda^{2}+m_{1} \lambda+m_{0}+\left(n_{2} \lambda^{2}+n_{1} \lambda+n_{0}\right) e^{-\lambda \tau_{1}} \\
& +\left(p_{1} \lambda+p_{0}\right) e^{-\lambda \tau_{2}}+q_{0} e^{-\lambda\left(\tau_{1}+\tau_{2}\right)}=0,
\end{aligned}
$$

where

$$
\begin{aligned}
& m_{0}=-a_{1} a_{4} a_{5}, \quad m_{1}=a_{1} a_{4}+a_{1} a_{5}+a_{4} a_{5}, \\
& m_{2}=-\left(a_{1}+a_{4}+a_{5}\right), \quad n_{0}=a_{5}\left(a_{1} b_{2}-a_{3} b_{1}\right), \\
& n_{1}=\left(a_{1}+a_{5}\right) b_{2}-a_{3} b_{1}, \quad n_{2}=-b_{2}, \\
& p_{0}=a_{2} a_{4} c_{1}, \quad p_{1}=-a_{2} c_{1}, \quad q_{0}=a_{2} b_{2} c_{1} .
\end{aligned}
$$

Case 1 $\left(\tau_{1}=\tau_{2}=0\right)$. Equation (8) becomes

$$
\lambda^{3}+m_{12} \lambda^{2}+m_{11} \lambda+m_{10}=0,
$$

where

$$
\begin{gathered}
m_{12}=m_{2}+n_{2}, \quad m_{11}=m_{1}+n_{1}+p_{1}, \\
m_{10}=m_{0}+n_{0}+p_{0}+q_{0}, \\
A_{10}=A_{0}+B_{0}+C_{0}+D_{0}+E_{0}+F_{0} .
\end{gathered}
$$

It follows from the Routh-Hurwitz criteria that all roots of (10) have negative real parts if the following condition holds: $\left(H_{11}\right): m_{12}>0$ and $m_{12} m_{11}>m_{10}$. Then, the positive equilibrium of system (2) without delay is locally asymptotically stable. 
Case $2\left(\tau_{1}>0, \tau_{2}=0\right)$. On substituting $\tau_{2}=0$, (8) becomes

$$
\lambda^{3}+m_{22} \lambda^{2}+m_{21} \lambda+m_{20}+\left(n_{22} \lambda^{2}+n_{21} \lambda+n_{20}\right) e^{-\lambda \tau_{1}}=0
$$

where

$$
\begin{gathered}
m_{22}=m_{2}, \quad m_{21}=m_{1}+p_{1}, \quad m_{20}=m_{0}+p_{0}, \\
n_{22}=n_{2}, \quad n_{21}=n_{1}, \quad n_{20}=n_{0}+q_{0} .
\end{gathered}
$$

Let $\lambda=i \omega_{1}\left(\omega_{1}>0\right)$ be a root of (12). Then, we have

$$
\begin{gathered}
n_{21} \omega_{1} \sin \omega_{1} \tau_{1}+\left(n_{20}-n_{22} \omega_{1}^{2}\right) \cos \omega_{1} \tau_{1}=m_{22} \omega_{1}^{2}-m_{20} \\
n_{21} \omega_{1} \cos \omega_{1} \tau_{1}-\left(n_{20}-n_{22} \omega_{1}^{2}\right) \sin \omega_{1} \tau_{1}=\omega_{1}^{3}-m_{21} \omega_{1}
\end{gathered}
$$

which implies that

$$
\omega_{1}^{6}+g_{22} \omega_{1}^{4}+g_{21} \omega_{1}^{2}+g_{20}=0
$$

where

$$
\begin{gathered}
g_{20}=m_{20}^{2}-n_{20}^{2}, \\
g_{21}=m_{21}^{2}-n_{21}^{2}-2 m_{20} m_{22}+2 n_{20} n_{22}, \\
g_{22}=m_{22}^{2}-n_{22}^{2}-2 m_{21} .
\end{gathered}
$$

Denote $\omega_{1}^{2}=v_{1}$; then (15) becomes

$$
v_{1}^{3}+g_{22} v_{1}^{2}+g_{21} v_{1}+g_{20}=0
$$

Let

$$
f_{1}\left(v_{1}\right)=v_{1}^{3}+g_{22} v_{1}^{2}+g_{21} v_{1}+g_{20} \text {. }
$$

In [17], Song et al. obtained the following results on the distribution of roots of (17).

Lemma 1. For (17),

(1) if $g_{20}<0$, then (17) has at least one positive root;

(2) if $g_{20} \geq 0$ and $g_{22}^{2}-3 g_{21} \leq 0$, then (17) has no positive roots;

(3) if $g_{20} \geq 0$ and $g_{22}^{2}-3 g_{21}<0$, then (17) has positive root if and only if $v_{1}^{*}=\left(-g_{20}+\sqrt{g_{22}^{2}+3 g_{21}}\right) / 3>0$ and $f_{1}\left(v_{1}^{*}\right) \leq 0$.

$\left(H_{21}\right)$ Suppose that $(17)$ has at least one positive root.

Without loss of generality, we assume that (17) has three positive roots, which are denoted by $v_{11}, v_{12}$, and $v_{13}$. Then (15) has three positive roots $\omega_{1 k}=\sqrt{v_{1 k}}, k=1,2,3$. For every fixed $\omega_{1 k}$, one can get

$$
\begin{aligned}
\sin \left(\omega_{1 k} \tau_{1}\right)= & \left(n_{22} \omega_{1 k}^{5}+\left(m_{22} n_{21}-m_{21} n_{22}-n_{20}\right) \omega_{1 k}^{3}\right. \\
& \left.\quad+\left(m_{21} n_{20}-m_{20} n_{21}\right) \omega_{1 k}\right) \\
& \times\left(n_{22}^{2} \omega_{1 k}^{4}+\left(n_{21}^{2}-2 n_{20} n_{22}\right) \omega_{1 k}^{2}+n_{20}^{2}\right)^{-1} \\
\triangleq & T_{2 s}\left(\omega_{1 k}\right), \\
\cos \left(\omega_{1 k} \tau_{1}\right)= & \left(\left(n_{21}-m_{22} n_{22}\right) \omega_{1 k}^{4}\right. \\
& +\left(m_{20} n_{22}+m_{22} n_{20}-m_{21} n_{21}\right) \\
& \left.\times \omega_{1 k}^{2}-m_{20} n_{20}\right) \\
& \times\left(n_{22}^{2} \omega_{1 k}^{4}+\left(n_{21}^{2}-2 n_{20} n_{22}\right) \omega_{1 k}^{2}+n_{20}^{2}\right)^{-1} \\
\triangleq & T_{2 c}\left(\omega_{1 k}\right) .
\end{aligned}
$$

Thus,

$\tau_{1 k}^{(j)}$

$$
= \begin{cases}\frac{1}{\omega_{1 k}}\left(\arccos \left(T_{2 c}\left(\omega_{1 k}\right)\right)+2 j \pi\right), & T_{2 s}\left(\omega_{1 k}\right) \geq 0, \\ \frac{1}{\omega_{1 k}}\left(2 \pi-\arccos \left(T_{2 c}\left(\omega_{1 k}\right)\right)+2 j \pi\right), & T_{2 s}\left(\omega_{1 k}\right)<0,\end{cases}
$$

with $k=1,2,3 ; j=0,1,2, \ldots$.

Let

$$
\begin{gathered}
\tau_{10}=\min \left\{\tau_{1 k}^{(0)}\right\}, \quad k=1,2,3, \\
\omega_{10}=\omega_{1 k_{0}} .
\end{gathered}
$$

Differentiating both sides of (12) with respect to $\tau_{1}$ we can get

$$
\begin{aligned}
{\left[\frac{d \lambda}{d \tau_{1}}\right]^{-1}=} & -\frac{3 \lambda^{2}+2 m_{22} \lambda+m_{21}}{\lambda\left(\lambda^{3}+m_{22} \lambda^{2}+m_{21} \lambda+m_{20}\right)} \\
& +\frac{2 n_{22} \lambda+n_{21}}{\lambda\left(n_{22} \lambda^{2}+n_{21} \lambda+n_{20}\right)}-\frac{\tau_{1}}{\lambda}
\end{aligned}
$$

Thus, we have

$$
\operatorname{Re}\left[\frac{d \lambda}{d \tau_{1}}\right]_{\tau=\tau_{10}}^{-1}=\frac{f_{1}^{\prime}\left(v_{1 *}\right)}{n_{22}^{2} \omega_{10}^{4}+\left(n_{21}^{2}-2 n_{20} n_{22}\right) \omega_{10}^{2}+n_{20}^{2}},
$$

where $v_{1 *}=\omega_{10}^{2}$. Obviously, if the condition $\left(H_{22}\right): f_{1}^{\prime}\left(v_{1 *}\right) \neq 0$ holds, then $\operatorname{Re}\left[d \lambda / d \tau_{1}\right]_{\tau_{1}=\tau_{10}}^{-1} \neq 0$. By the Hopf bifurcation theorem in [18], we have the following results.

Theorem 2. Suppose that conditions $\left(H_{21}\right)-\left(H_{22}\right)$ hold. The positive equilibrium $E^{*}\left(S^{*}, I^{*}, P^{*}\right)$ of system (2) is asymptotically stable for $\tau_{1} \in\left[0, \tau_{10}\right)$ and system (2) undergoes a Hopf bifurcation at $E^{*}\left(S^{*}, I^{*}, P^{*}\right)$ when $\tau_{1}=\tau_{10}$. 
Case $3\left(\tau_{2}>0, \tau_{1}=0\right)$. Substitute $\tau_{1}=0$ into (8), then (8) becomes

$$
\lambda^{3}+m_{32} \lambda^{2}+m_{31} \lambda+m_{30}+\left(p_{31} \lambda+p_{30}\right) e^{-\lambda \tau_{2}}=0,
$$

where

$$
\begin{gathered}
m_{32}=m_{2}+n_{2}, \quad m_{31}=m_{1}+n_{1}, \\
m_{30}=m_{0}+n_{0}, \quad p_{31}=p_{1}, \\
p_{30}=p_{0}+q_{0} .
\end{gathered}
$$

Let $\lambda=i \omega_{2}\left(\omega_{2}>0\right)$ be a root of (24). Then, we get

$$
\begin{gathered}
p_{31} \omega_{2} \sin \omega_{2} \tau_{2}+p_{30} \cos \omega_{2} \tau_{2}=m_{32} \omega_{2}^{2}-m_{30}, \\
p_{31} \omega_{2} \cos \omega_{2} \tau_{2}-p_{30} \sin \omega_{2} \tau_{2}=\omega_{2}^{3}-m_{31} \omega_{2},
\end{gathered}
$$

which follows that

$$
\lambda_{2}^{6}+g_{32} \lambda_{2}^{4}+g_{31} \lambda_{2}+g_{30}=0
$$

where

$$
\begin{gathered}
g_{32}=m_{32}^{2}-2 m_{31}, \quad g_{31}=m_{31}^{2}-2 m_{30} m_{32}-p_{31}^{2}, \\
g_{30}=m_{30}^{2}-p_{30}^{2} .
\end{gathered}
$$

Denote $\omega_{2}^{2}=v_{2}$; then (27) becomes

$$
v_{2}^{3}+g_{32} v_{2}^{2}+g_{31} v_{2}+g_{30}=0
$$

Let

$$
f_{2}\left(v_{2}\right)=v_{2}^{3}+g_{32} v_{2}^{2}+g_{31} v_{2}+g_{30}=0
$$

Similarly as in Case 2, we suppose that $\left(H_{31}\right)$, (29) has at least one positive root. Without loss of generality, we assume that it has three positive roots and we denote them by $v_{21}$, $v_{22}$, and $v_{23}$, respectively. Then (27) has three positive roots $\omega_{2 k}=\sqrt{v_{2 k}}, k=1,2,3$. For every fixed $\omega_{2 k}$,

$$
\begin{aligned}
& \sin \left(\omega_{2 k} \tau_{2}\right) \\
& =\frac{\left(m_{32} p_{31}-p_{30}\right) \omega_{2 k}^{3}+\left(m_{31} p_{30}-m_{30} p_{31}\right) \omega_{2 k}}{p_{31}^{2} \omega_{2 k}^{2}+p_{30}^{2}} \\
& \quad \triangleq T_{3 s}\left(\omega_{2 k}\right), \\
& \cos \left(\omega_{2 k} \tau_{2}\right) \\
& \quad=\frac{p_{31} \omega_{2 k}^{4}+\left(m_{32} p_{30}-m_{31} p_{31}\right) \omega_{2 k}^{2}-m_{30} p_{30}}{p_{31}^{2} \omega_{2 k}^{2}+p_{30}^{2}} \\
& \triangleq T_{3 c}\left(\omega_{2 k}\right) .
\end{aligned}
$$

Thus,

$$
\begin{aligned}
& \tau_{2 k}^{(j)} \\
& = \begin{cases}\frac{1}{\omega_{2 k}}\left(\arccos \left(T_{3 c}\left(\omega_{2 k}\right)\right)+2 j \pi\right), & T_{3 s}\left(\omega_{2 k}\right) \geq 0, \\
\frac{1}{\omega_{2 k}}\left(2 \pi-\arccos \left(T_{3 c}\left(\omega_{2 k}\right)\right)+2 j \pi\right), & T_{3 s}\left(\omega_{2 k}\right)<0,\end{cases}
\end{aligned}
$$

with $k=1,2,3 ; j=0,1,2, \ldots$.
Let

$$
\begin{gathered}
\tau_{20}=\min \left\{\tau_{2 k}^{(0)}\right\}, \quad k=1,2,3, \\
\omega_{20}=\omega_{2 k_{0}} .
\end{gathered}
$$

Differentiating (24) regarding $\tau_{2}$, we get

$$
\begin{aligned}
{\left[\frac{d \lambda}{d \tau_{2}}\right]^{-1}=} & \frac{3 \lambda^{2}+2 m_{32} \lambda+m_{31}}{\lambda\left(\lambda^{3}+m_{32} \lambda^{2}+m_{31} \lambda+m_{30}\right)} \\
& +\frac{p_{31}}{\lambda\left(p_{31} \lambda+p_{30}\right)}-\frac{\tau_{2}}{\lambda} .
\end{aligned}
$$

Then, we can get

$$
\operatorname{Re}\left[\frac{d \lambda}{d \tau_{2}}\right]_{\tau=\tau_{20}}^{-1}=\frac{f_{2}^{\prime}\left(v_{2 *}\right)}{p_{31}^{2} \omega_{20}^{2}+p_{30}^{2}},
$$

where $v_{2 *}=\omega_{20}^{2}$. Therefore, if the condition $\left(H_{32}\right): f_{2}^{\prime}\left(v_{2 *}\right) \neq 0$ holds, then $\operatorname{Re}\left[d \lambda / d \tau_{2}\right]_{\tau_{2}=\tau_{20}}^{-1} \neq 0$. Thus, by the Hopf bifurcation theorem in [18], we have the following results.

Theorem 3. Suppose that conditions $\left(H_{31}\right)-\left(H_{32}\right)$ hold. The positive equilibrium $E^{*}\left(S^{*}, I^{*}, P^{*}\right)$ of system (2) is asymptotically stable for $\tau_{1} \in\left[0, \tau_{20}\right)$ and system (2) undergoes a Hopf bifurcation at $E^{*}\left(S^{*}, I^{*}, P^{*}\right)$ when $\tau_{2}=\tau_{20}$.

Case $4\left(\tau_{1}=\tau_{2}=\tau>0\right)$. Let $\tau_{1}=\tau_{2}=\tau$; then (8) becomes

$$
\begin{aligned}
\lambda^{3} & +m_{42} \lambda^{2}+m_{41} \lambda+m_{40}+\left(n_{42} \lambda^{2}+n_{41} \lambda+n_{40}\right) e^{-\lambda \tau} \\
& +q_{40} e^{-2 \lambda \tau}=0,
\end{aligned}
$$

where

$$
\begin{gathered}
m_{42}=m_{2}, \quad m_{41}=m_{1}, \quad m_{40}=m_{0}, \quad n_{42}=n_{2}, \\
n_{41}=n_{1}+p_{1}, \quad n_{40}=n_{0}+p_{0}, \quad q_{40}=q_{0} .
\end{gathered}
$$

Multiplying (37) by $e^{\lambda \tau}$, then (37) becomes

$$
\begin{aligned}
n_{42} \lambda^{2}+ & n_{41} \lambda+n_{40}+\left(\lambda^{3}+m_{42} \lambda^{2}+m_{41} \lambda+m_{40}\right) e^{\lambda \tau} \\
& +q_{40} e^{-\lambda \tau}=0 .
\end{aligned}
$$

Let $\lambda=i \omega(\omega>0)$ be the root of (39); then we can get

$$
\begin{aligned}
& \left(m_{40}+q_{40}-m_{42} \omega^{2}\right) \cos \tau \omega+\left(\omega^{3}-m_{41} \omega\right) \sin \tau \omega \\
& \quad=n_{32} \omega^{2}-n_{40}, \\
& \left(m_{40}-q_{40}-m_{42} \omega^{2}\right) \sin \tau \omega-\left(\omega^{3}-m_{41} \omega\right) \cos \tau \omega \\
& =-n_{41} \omega,
\end{aligned}
$$

which follows that

$$
\begin{aligned}
& \sin (\tau \omega)=\frac{g_{5} \omega^{5}+g_{3} \omega^{3}+g_{1} \omega}{\omega^{6}+h_{4} \omega^{4}+h_{2} \omega^{2}+h_{0}} \triangleq T_{4 s}(\omega), \\
& \cos (\tau \omega)=\frac{g_{4} \omega^{4}+g_{2} \omega^{2}+g_{0}}{\omega^{6}+h_{4} \omega^{4}+h_{2} \omega^{2}+h_{0}} \triangleq T_{4 c}(\omega),
\end{aligned}
$$


where

$$
\begin{gathered}
g_{0}=n_{40} q_{40}-m_{40} n_{40}, \\
g_{1}=m_{41} n_{40}-m_{40} n_{41}-n_{41} q_{40}, \\
g_{2}=m_{40} n_{42}-m_{41} n_{41}+m_{42} n_{40}-n_{42} q_{40}, \\
g_{3}=m_{42} n_{41}-m_{41} n_{42}-n_{40}, \\
g_{4}=n_{41}-m_{42} n_{42}, \quad g_{5}=n_{42}, \quad h_{0}=m_{40}^{2}-q_{40}^{2}, \\
h_{2}=m_{41}^{2}-2 m_{40} m_{42}, \quad h_{4}=m_{42}^{2}-2 m_{41} .
\end{gathered}
$$

Then, we can obtain

$$
\omega^{12}+g_{45} \omega^{10}+g_{44} \omega^{8}+g_{43} \omega^{6}+g_{42} \omega^{4}+g_{41} \omega^{2}+g_{40}=0,
$$

where

$$
\begin{gathered}
g_{40}=h_{0}^{2}-g_{0}^{2}, \quad g_{41}=2 h_{0} h_{2}-2 g_{0} g_{2}-g_{1}^{2}, \\
g_{42}=h_{2}^{2}+2 h_{0} h_{4}-g_{2}^{2}-2 g_{0} g_{4}-2 g_{1} g_{3}, \\
g_{43}=2 h_{0}+2 h_{2} h_{4}-g_{3}^{2}-2 g_{1} g_{5}-2 g_{2} g_{4}, \\
g_{44}=h_{4}^{2}+2 h_{2}-g_{4}^{2}-2 g_{3} g_{5}, \quad g_{45}=2 h_{4}-g_{5}^{2} .
\end{gathered}
$$

Let $\omega^{2}=v_{3}$; then (43) becomes

$$
v_{3}^{6}+g_{45} v_{3}^{5}+g_{44} v_{3}^{4}+g_{43} v_{3}^{3}+g_{42} v_{3}^{2}+g_{41} v_{3}+g_{40}=0 .
$$

If we know all the coefficients of system (2), then we can get all the coefficients of (45) and then all the roots of (45) can be obtained by Matlab. Therefore, we give the following assumption.

Suppose that $\left(H_{41}\right):(45)$ has at least one positive root.

Without loss of generality, we assume that (45) has six positive roots, which are denoted by $v_{31}, v_{32}, \ldots, v_{36}$, respectively. Then, (43) has six positive roots $\omega_{k}=\sqrt{v_{3 k}}$, $k=1,2, \ldots, 6$. For every $\omega_{k}$,

$$
\begin{aligned}
& \tau_{k}^{(j)} \\
& = \begin{cases}\frac{1}{\omega_{k}}\left(\arccos \left(T_{4 c}\left(\omega_{k}\right)\right)+2 j \pi\right), & T_{4 s}\left(\omega_{k}\right) \geq 0, \\
\frac{1}{\omega_{k}}\left(2 \pi-\arccos \left(T_{4 c}\left(\omega_{k}\right)\right)+2 j \pi\right), & T_{4 s}\left(\omega_{k}\right)<0,\end{cases}
\end{aligned}
$$

with $k=1,2,3, \ldots, 6 ; j=0,1,2, \ldots$.

Let

$$
\begin{gathered}
\tau_{0}=\min \left\{\tau_{k}^{(0)}\right\}, \quad k=1,2, \ldots, 6, \\
\omega_{0}=\omega_{k_{0}} .
\end{gathered}
$$

Next, taking the derivative of $\lambda$ with respect to $\tau$ in (39), we have

$$
\begin{aligned}
& {\left[\frac{d \lambda}{d \tau}\right]^{-1}=}\left(2 n_{42} \lambda+n_{41}+\left(3 \lambda^{2}+2 m_{42} \lambda+m_{41}\right) e^{\lambda \tau}\right) \\
& \times\left(q_{40} \lambda e^{-\lambda \tau}-\left(\lambda^{4}+m_{42} \lambda^{3}+m_{41} \lambda^{2}\right.\right. \\
&\left.\left.+m_{40} \lambda\right) e^{\lambda \tau}\right)^{-1}-\frac{\tau}{\lambda}
\end{aligned}
$$

Then we have

$$
\operatorname{Re}\left[\frac{d \lambda}{d \tau}\right]_{\tau=\tau_{0}}^{-1}=\frac{P_{R} Q_{R}+P_{I} Q_{I}}{Q_{R}^{2}+Q_{I}^{2}},
$$

where

$$
\begin{gathered}
P_{R}=\left(m_{41}-3 \omega_{0}^{2}\right) \cos \tau_{0} \omega_{0}-2 m_{42} \omega_{0} \sin \tau_{0} \omega_{0}+n_{41}, \\
P_{I}=\left(m_{41}-3 \omega_{0}^{2}\right) \sin \tau_{0} \omega_{0}+2 m_{42} \omega_{0} \cos \tau_{0} \omega_{0}+2 n_{42} \omega_{0}, \\
Q_{R}=\left(m_{41} \omega_{0}^{2}-\omega_{0}^{4}\right) \cos \tau_{0} \omega_{0} \\
\\
\quad-\left(m_{42} \omega_{0}^{3}-m_{40} \omega_{0}-q_{40} \omega_{0}\right) \sin \tau_{0} \omega_{0}, \\
Q_{I}=\left(m_{41} \omega_{0}^{2}-\omega_{0}^{4}\right) \sin \tau_{0} \omega_{0} \\
+ \\
+\left(m_{42} \omega_{0}^{3}-m_{40} \omega_{0}+q_{40} \omega_{0}\right) \cos \tau_{0} \omega_{0} .
\end{gathered}
$$

Obviously, if condition $\left(H_{42}\right): P_{R} Q_{R}+P_{I} Q_{I} \neq 0$ holds, then $\operatorname{Re}[d \lambda / d \tau]_{\tau=\tau_{0}}^{-1} \neq 0$. Thus, by the discussion above and the Hopf bifurcation theorem in [18], we have the following results.

Theorem 4. Suppose that conditions $\left(H_{41}\right)-\left(H_{42}\right)$ hold. The positive equilibrium $E^{*}\left(S^{*}, I^{*}, P^{*}\right)$ of system (2) is asymptotically stable for $\tau_{1} \in\left[0, \tau_{0}\right)$ and system (2) undergoes a Hopf bifurcation at $E^{*}\left(S^{*}, I^{*}, P^{*}\right)$ when $\tau=\tau_{0}$.

Case $5\left(\tau_{2}>0\right.$ and $\left.\tau_{1} \in\left(0, \tau_{10}\right)\right)$. Let $\lambda=i \omega_{2}^{*}\left(\omega_{2}^{*}\right)$ be the root of (8). Then, we get

$$
\begin{aligned}
& M_{51} \sin \tau_{2} \omega_{2}^{*}+M_{52} \cos \tau_{2} \omega_{2}^{*}=N_{51}, \\
& M_{51} \cos \tau_{2} \omega_{2}^{*}-M_{52} \sin \tau_{2} \omega_{2}^{*}=N_{52},
\end{aligned}
$$

where

$$
M_{51}=p_{1} \omega_{2}^{*}-q_{0} \sin \tau_{1} \omega_{2}^{*}, \quad N_{52}=p_{0}+q_{0} \cos \tau_{1} \omega_{2}^{*},
$$

$$
\begin{aligned}
N_{51}= & m_{2}\left(\omega_{2}^{*}\right)^{2}-m_{0}-\left(n_{0}-n_{2}\left(\omega_{2}^{*}\right)^{2}\right) \cos \tau_{1} \omega_{2}^{*} \\
& -n_{1} \omega_{2}^{*} \sin \tau_{1} \omega_{2}^{*}, \\
N_{52}= & \left(\omega_{2}^{*}\right)^{3}-m_{1} \omega_{2}^{*}+\left(n_{0}-n_{2}\left(\omega_{2}^{*}\right)^{2}\right) \sin \tau_{1} \omega_{2}^{*} \\
& -n_{1} \omega_{2}^{*} \cos \tau_{1} \omega_{2}^{*} .
\end{aligned}
$$



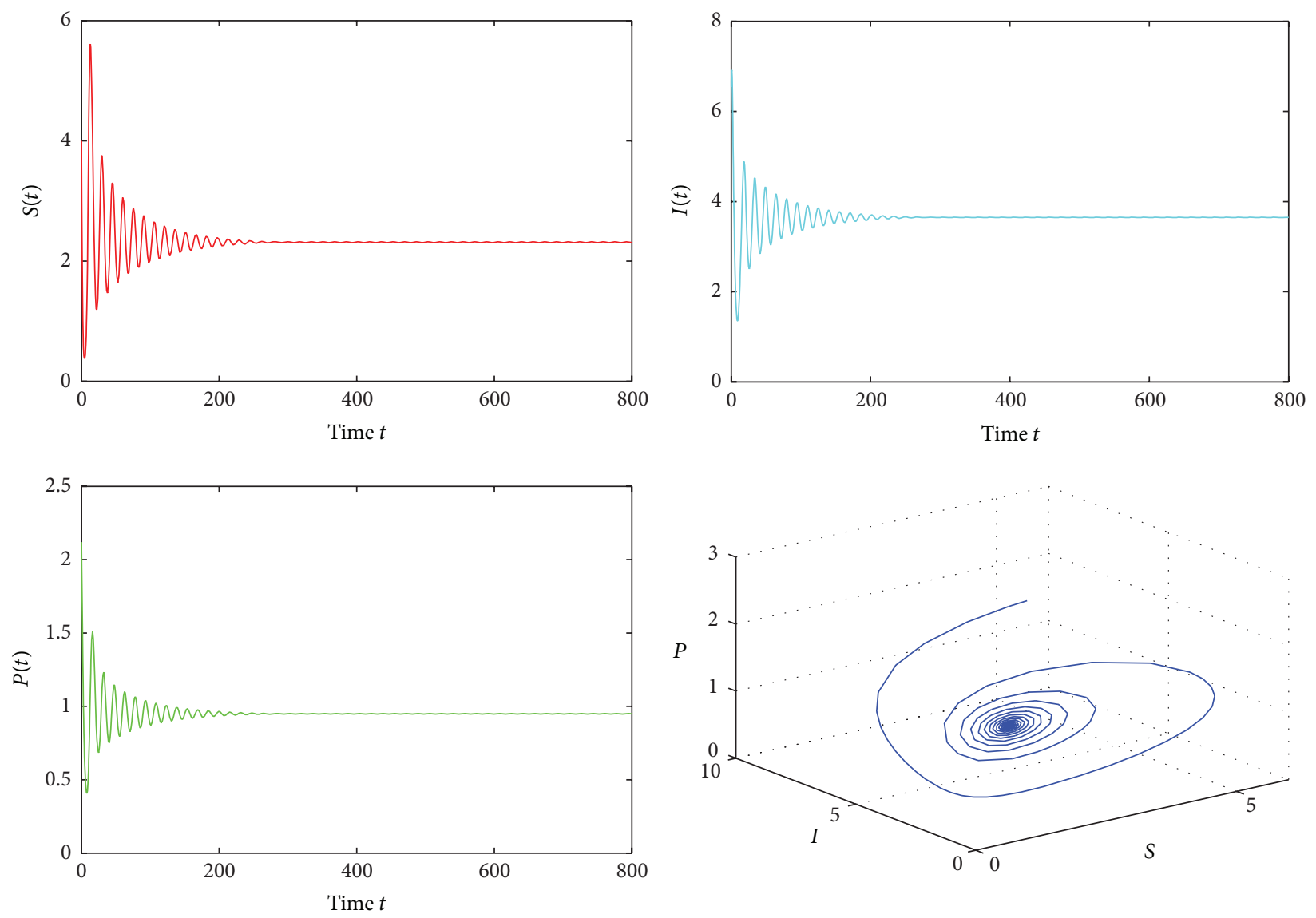

FIGURE 1: $E^{*}$ is asymptotically stable when $\tau_{1}=0.45<0.5148=\tau_{10}$.

Then, we can have

$$
g_{51}\left(\omega_{2}^{*}\right)+2 g_{52}\left(\omega_{2}^{*}\right) \cos \tau_{1} \omega_{2}^{*}+2 g_{53}\left(\omega_{2}^{*}\right) \sin \tau_{1} \omega_{2}^{*}=0 \text {, }
$$

where

$$
\begin{aligned}
g_{51}\left(\omega_{2}^{*}\right)= & \left(\omega_{2}^{*}\right)^{6}+\left(m_{2}^{2}+n_{2}^{2}-2 m_{1}\right)\left(\omega_{2}^{*}\right)^{4} \\
& +\left(m_{1}^{2}+n_{1}^{2}-p_{1}^{2}-2 m_{0} m_{2}-2 n_{0} n_{2}\right)\left(\omega_{2}^{*}\right)^{2} \\
& +m_{0}^{2}+n_{0}^{2}-p_{0}^{2}-q_{0}^{2}, g_{52}\left(\omega_{2}^{*}\right) \\
= & \left(m_{2} n_{2}-n_{1}\right)\left(\omega_{2}^{*}\right)^{4} \\
& +\left(m_{1} n_{1}-m_{0} n_{2}-m_{2} n_{0}\right)\left(\omega_{2}^{*}\right)^{2} \\
& +m_{0} n_{0}-p_{0} q_{0}, g_{53}\left(\omega_{2}^{*}\right) \\
= & -n_{2}\left(\omega_{2}^{*}\right)^{5}+\left(n_{0}-m_{2} n_{1}+m_{1} n_{2}\right)\left(\omega_{2}^{*}\right)^{3} \\
& +\left(m_{0} n_{1}-m_{1} n_{0}+p_{1} q_{0}\right) \omega_{2}^{*} .
\end{aligned}
$$

We suppose that $\left(H_{51}\right)$, (55) has at least finite positive roots. And we denote the positive roots of (55) by $\omega_{21}^{*}, \omega_{22}^{*}, \ldots, \omega_{2 k}^{*}$. Then, for every fixed $\omega_{2 i}^{*}(i=1,2, \ldots, k)$,

$$
\begin{aligned}
& \sin \left(\tau_{2} \omega_{2 i}^{*}\right)=\left.\frac{M_{51} N_{51}-M_{52} N_{52}}{M_{51}^{2}+M_{52}^{2}}\right|_{\omega_{2}^{*}=\omega_{2 i}^{*}} \triangleq T_{5 s}\left(\omega_{2 i}^{*}\right), \\
& \cos \left(\tau_{2} \omega_{2 i}^{*}\right)=\left.\frac{M_{51} N_{52}+M_{52} N_{51}}{M_{51}^{2}+M_{52}^{2}}\right|_{\omega_{2}^{*}=\omega_{2 i}^{*}} \triangleq T_{5 c}\left(\omega_{2 i}^{*}\right) .
\end{aligned}
$$

Thus,

$$
\begin{aligned}
& \tau_{2 i}^{*(j)} \\
& = \begin{cases}\frac{1}{\omega_{2 i}^{*}}\left(\arccos \left(T_{5 c}\left(\omega_{2 i}^{*}\right)\right)+2 j \pi\right), & T_{5 s}\left(\omega_{k}\right) \geq 0, \\
\frac{1}{\omega_{2 i}^{*}}\left(2 \pi-\arccos \left(T_{5 c}\left(\omega_{2 i}^{*}\right)\right)+2 j \pi\right), & T_{5 s}\left(\omega_{k}\right)<0,\end{cases}
\end{aligned}
$$

with $i=1,2, \ldots, k ; j=0,1,2, \ldots$

Let $\tau_{20}^{*}=\min \left\{\tau_{2 i}^{*(0)} \mid i=1,2, \ldots, k\right\}$. When $\tau_{2}=\tau_{20}^{*},(8)$ has a pair of purely imaginary roots $\pm i \omega_{2 *}$ for $\tau_{1} \in\left(0, \tau_{10}\right)$. Next, in order to give the main results with respect to $\tau_{2}>$ $0, \tau_{1} \in\left(0, \tau_{10}\right)$, we give the following assumption: $\left(H_{52}\right)$ : $\operatorname{Re}\left[d \lambda / d \tau_{2}\right]_{\tau=\tau_{20}^{*}}^{-1} \neq 0$. 

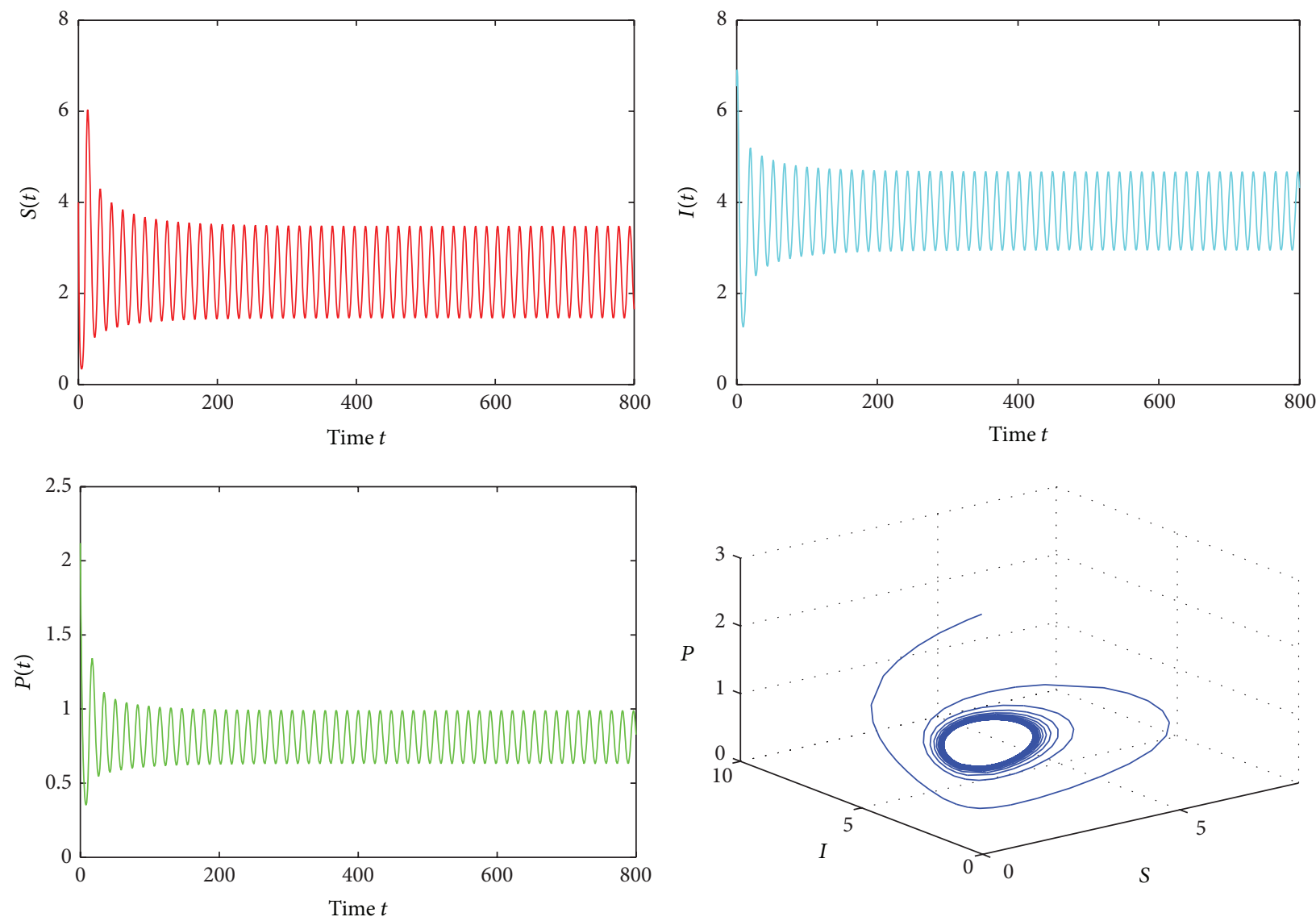

FIgURE 2: $E^{*}$ is unstable when $\tau_{1}=0.575>0.5148=\tau_{10}$.

Through the analysis above and the Hopf bifurcation theorem in [18], we have the following results.

Theorem 5. If conditions $\left(H_{51}\right)-\left(H_{52}\right)$ hold and $\tau_{1} \in\left(0, \tau_{10}\right)$, then the positive equilibrium $E^{*}\left(S^{*}, I^{*}, P^{*}\right)$ of system (2) is asymptotically stable for $\tau_{2} \in\left[0, \tau_{20}^{*}\right)$ and system (2) undergoes a Hopf bifurcation at $E^{*}\left(S^{*}, I^{*}, P^{*}\right)$ when $\tau_{2}=\tau_{20}^{*}$.

\section{Stability of Bifurcating Periodic Solutions}

In the previous section, it is shown that system (2) undergoes a Hopf bifurcation for different combinations of $\tau_{1}$ and $\tau_{2}$ under certain conditions. In this section, the properties of Hopf bifurcation such as direction and stability are investigated with respect to $\tau_{2}$ for $\tau_{1} \in\left(0, \tau_{10}\right)$ by using the normal form method and center manifold theorem in [18]. Throughout this section, we assume that $\tau_{10}^{*}<\tau_{20}^{*}$ where $\tau_{10}^{*} \in\left(0, \tau_{10}\right)$.

For convenience, let $\tau_{2}=\mu+\tau_{20}^{*}$, so that $\mu=0$ is the Hopf bifurcation value of system (2). Let $u_{1}(t)=S(t)-S^{*}$, $u_{2}(t)=I(t)-I^{*}, u_{3}(t)=P(t)-P^{*}$ and rescale the time delay $t \rightarrow\left(t / \tau_{2}\right)$; then system (2) can be rewritten as

$$
\dot{u}(t)=L_{\mu} u_{t}+F\left(\mu, u_{t}\right),
$$

where

$$
\begin{gathered}
L_{\mu} \phi=\left(\tau_{20}^{*}+\mu\right)\left(A^{\prime} \phi(0)+B^{\prime} \phi\left(-\frac{\tau_{10}^{*}}{\tau_{20}^{*}}\right)+C^{\prime} \phi(-1)\right), \\
F(\mu, \phi)=\left(\tau_{20}^{*}+\mu\right)\left(F_{1}, F_{2}, F_{3}\right)^{T},
\end{gathered}
$$

with

$$
\begin{gathered}
\phi(\theta)=\left(\phi_{1}(\theta), \phi_{2}(\theta), \phi_{3}(\theta)\right)^{T} \in C\left([-1,0], R^{3}\right), \\
A^{\prime}=\left(\begin{array}{ccc}
a_{1} & 0 & a_{2} \\
a_{3} & a_{4} & 0 \\
0 & 0 & a_{5}
\end{array}\right), \quad B^{\prime}=\left(\begin{array}{ccc}
0 & b_{1} & 0 \\
0 & b_{2} & 0 \\
0 & 0 & 0
\end{array}\right), \\
C^{\prime}=\left(\begin{array}{lll}
0 & 0 & 0 \\
0 & 0 & 0 \\
c_{1} & 0 & 0
\end{array}\right), \\
F_{1}=a_{21} \phi_{1}^{2}(0)+a_{22} \phi_{1}(0) \phi_{2}\left(-\frac{\tau_{10}^{*}}{\tau_{20}^{*}}\right) \\
+a_{23} \phi_{1}(0) \phi_{3}(0)+a_{31} \phi_{1}^{3}(0) \\
+a_{32} \phi_{1}^{2}(0) \phi_{2}\left(-\frac{\tau_{10}^{*}}{\tau_{20}^{*}}\right)+a_{33} \phi_{1}^{2}(0) \phi_{3}(0)+\cdots, \\
F_{2}=b_{21} \phi_{1}^{2}(0)+b_{22} \phi_{1}(0) \phi_{2}\left(-\frac{\tau_{10}^{*}}{\tau_{20}^{*}}\right)
\end{gathered}
$$



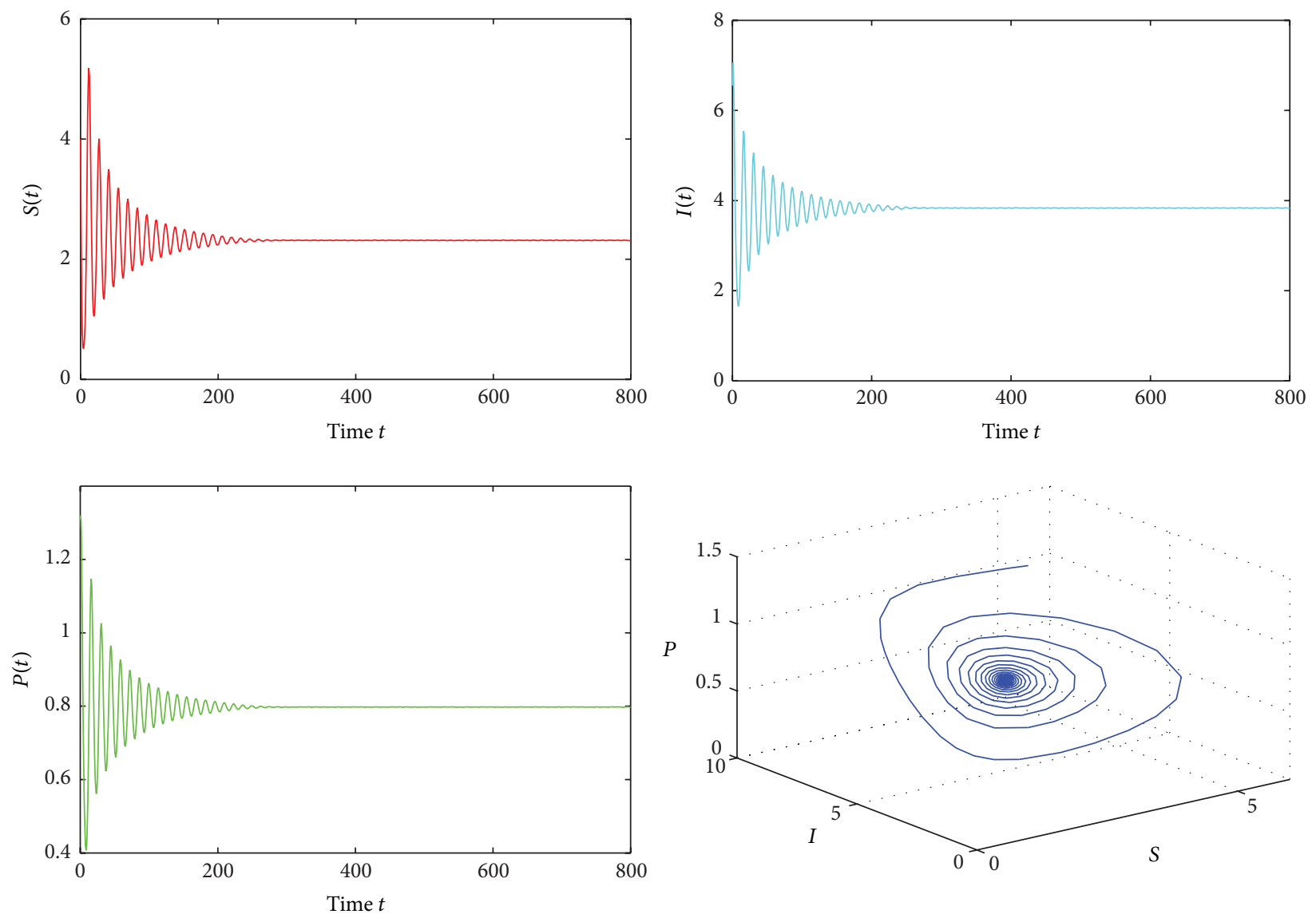

FIGURE 3: $E^{*}$ is asymptotically stable when $\tau_{2}=1.275<1.8829=\tau_{20}$.

$$
\begin{aligned}
& +b_{31} \phi_{1}^{3}(0)+b_{32} \phi_{1}^{2}(0) \phi_{2}\left(-\frac{\tau_{10}^{*}}{\tau_{20}^{*}}\right)+\cdots, \\
F_{3}= & c_{21} \phi_{3}^{2}(0)+c_{22} \phi_{1}(-1) \phi_{3}(0)+c_{23} \phi_{1}^{2}(-1) \\
& +c_{31} \phi_{1}^{2}(-1) \phi_{3}(0)+c_{32} \phi_{1}^{3}(-1)+\cdots .
\end{aligned}
$$

Therefore, according to the Riesz representation theorem, there exists a $3 \times 3$ matrix function $\eta(\theta, \mu):[-1,0] \rightarrow R^{3}$ whose elements are of bounded variation such that

$$
L_{\mu} \phi=\int_{-1}^{0} d \eta(\theta, \mu) \phi(\theta), \quad \phi \in C\left([-1,0], R^{3}\right) .
$$

In fact, we choose

$$
\eta(\theta, \mu)= \begin{cases}\left(\tau_{20}^{*}+\mu\right)\left(A^{\prime}+B^{\prime}+C^{\prime}\right), & \theta=0, \\ \left(\tau_{20}^{*}+\mu\right)\left(B^{\prime}+C^{\prime}\right), & \theta \in\left[-\frac{\tau_{10}^{*}}{\tau_{20}^{*}}, 0\right), \\ \left(\tau_{20}^{*}+\mu\right) C^{\prime}, & \theta \in\left(-1,-\frac{\tau_{10}^{*}}{\tau_{20}^{*}}\right), \\ 0, & \theta=-1 .\end{cases}
$$

For $\phi \in C\left([-1,0], R^{3}\right)$, we define

$$
\begin{gathered}
A(\mu) \phi= \begin{cases}\frac{d \phi(\theta)}{d \theta}, & -1 \leq \theta<0, \\
\int_{-1}^{0} d \eta(\theta, \mu) \phi(\theta), & \theta=0,\end{cases} \\
R(\mu) \phi= \begin{cases}0, & -1 \leq \theta<0, \\
F(\mu, \phi), & \theta=0 .\end{cases}
\end{gathered}
$$

Then system (59) can be transformed into the following operator equation:

$$
\dot{u}(t)=A(\mu) u_{t}+R(\mu) u_{t},
$$

where $u_{t}=u(t+\theta)=\left(u_{1}(t+\theta), u_{2}(t+\theta), u_{3}(t+\theta)\right)$ for $\theta \in[-1,0]$.

For $\varphi \in C^{1}\left([0,1],\left(R^{3}\right)^{*}\right)$, where $\left(R^{3}\right)^{*}$ is the 3-dimensional space of row vectors, we define the adjoint operator $A^{*}$ of $A$ :

$$
A^{*}(\varphi)= \begin{cases}-\frac{d \varphi(s)}{d s}, & 0<s \leq 1, \\ \int_{-1}^{0} d \eta^{T}(s, 0) \varphi(-s), & s=0,\end{cases}
$$



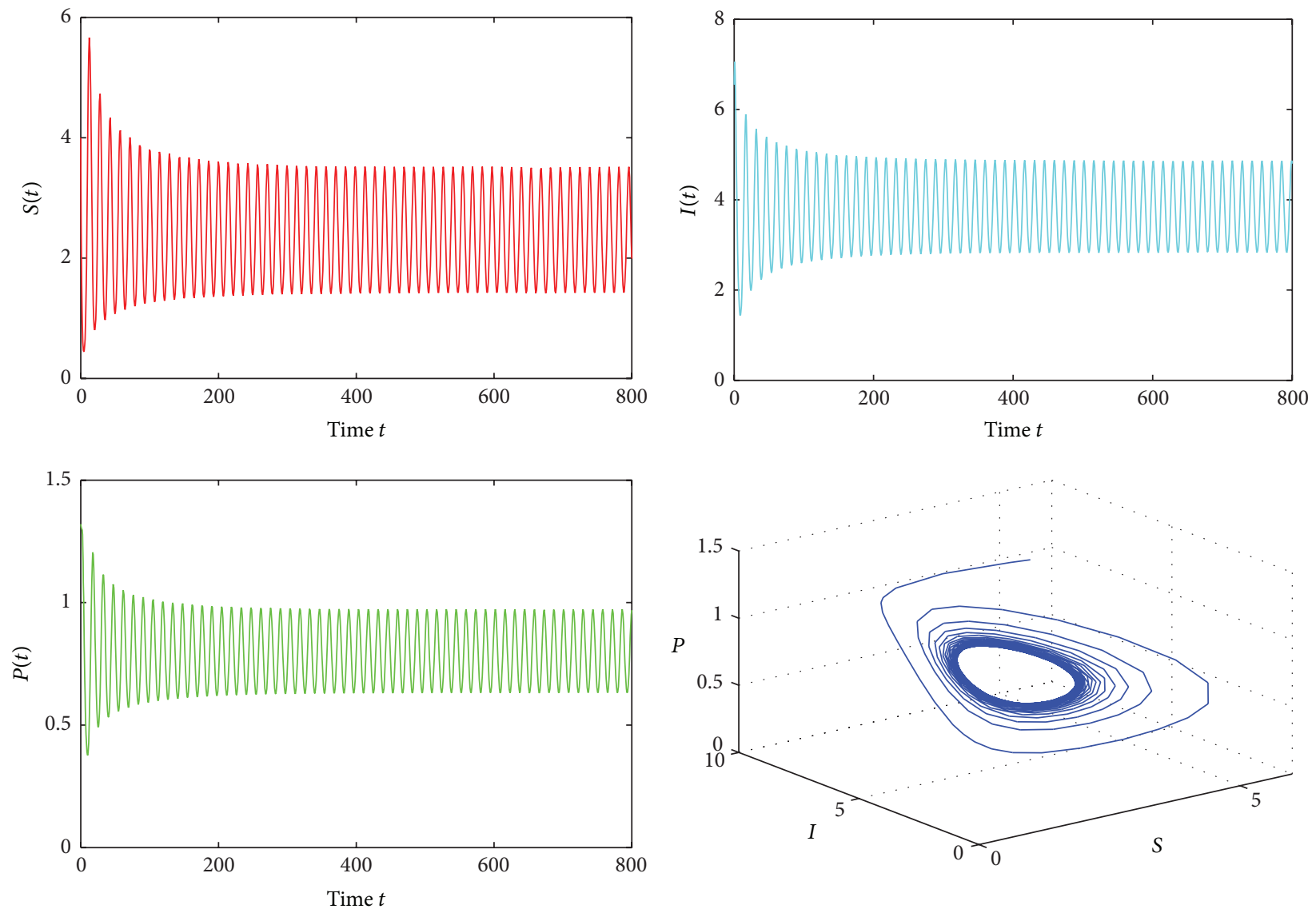

Figure 4: $E^{*}$ is unstable when $\tau_{2}=2.25>1.8829=\tau_{20}$.

and a bilinear inner product

$$
\begin{aligned}
\langle\varphi(s), \phi(\theta)\rangle= & \bar{\varphi}(0) \phi(0) \\
& -\int_{\theta=-1}^{0} \int_{\xi=0}^{\theta} \bar{\varphi}(\xi-\theta) d \eta(\theta) \phi(\xi) d \xi
\end{aligned}
$$

where $\eta(\theta)=\eta(\theta, 0)$.

Then $A(0)$ and $A^{*}(0)$ are adjoint operators. From the discussion above, we know that $\pm i \omega_{2 *} \tau_{20}^{*}$ are eigenvalues of $A(0)$ and they are also eigenvalues of $A^{*}(0)$.

Let $q(\theta)=\left(1, q_{2}, q_{3}\right)^{T} e^{i \omega_{2 *} \tau_{20}^{*} \theta}$ be the eigenvectors of $A(0)$ corresponding to the eigenvalue $+i \omega_{2 *} \tau_{20}^{*}$ and $q^{*}(s)=D(1$, $\left.q_{2}^{*}, q_{3}^{*}\right) e^{i \omega_{2 *} \tau_{20}^{*}} s$ the eigenvectors of $A^{*}(0)$ corresponding to the eigenvalue $-i \omega_{2 *} \tau_{20}^{*}$.

It is not difficult to verify that

$$
\begin{aligned}
& q_{2}=\frac{c_{1} e^{-i \omega_{2 *} \tau_{20}^{*}}}{i \omega_{2 *}-a_{5}}, \quad q_{3}=\frac{a_{3}}{i \omega_{2 *}-a_{4}-b_{2} e^{-i \omega_{2 *} \tau_{20}^{*}}}, \\
& q_{2}^{*}=-\frac{b_{1} e^{i \omega_{2 *} \tau_{20}^{*}}}{i \omega_{2 *}+a_{4}+b_{2} e^{i \omega_{2 *} \tau_{20}^{*}}}, \quad q_{3}^{*}=-\frac{a_{2}}{i \omega_{2 *}+a_{5}} .
\end{aligned}
$$

From (67), we can get

$$
\begin{aligned}
\bar{D}=\left[1+q_{2} \bar{q}_{2}^{*}+q_{3} \bar{q}_{3}^{*}\right. & +b_{11} \tau_{10}^{*} e^{-i \omega_{1}^{*} \tau_{10}^{*}} \\
+ & \tau_{2}^{*} e^{-i \omega_{1}^{*} \tau_{2 *}}\left(\bar{q}_{2}^{*}\left(c_{21}+c_{22} q_{2}\right)\right. \\
& \left.\left.+\bar{q}_{3}^{*}\left(c_{32} q_{2}+c_{33} q_{3}\right)\right)\right]^{-1},
\end{aligned}
$$

such that $\left\langle q^{*}, q\right\rangle=1,\left\langle q^{*}, \bar{q}\right\rangle=0$.

In the remainder of this section, we obtain the coefficients used to determine the properties of the periodic solution by the algorithms given in [18] and using a computation process similar to that in [11]:

$$
\begin{gathered}
g_{20}=2 \tau_{20}^{*} \bar{D}\left[a_{21}+a_{22} q^{(2)}\left(-\frac{\tau_{10}^{*}}{\tau_{20}^{*}}\right)+a_{23} q^{(3)}(0)\right. \\
+\bar{q}_{2}^{*}\left(b_{21}+b_{22} q^{(2)}\left(-\frac{\tau_{10}^{*}}{\tau_{20}^{*}}\right)\right) \\
+\bar{q}_{3}^{*}\left(c_{21}\left(q^{(3)}(0)\right)^{2}+c_{22} q^{(1)}(-1) q^{(3)}(0)\right. \\
\left.\left.+c_{23}\left(q^{(1)}(-1)\right)^{2}\right)\right]
\end{gathered}
$$



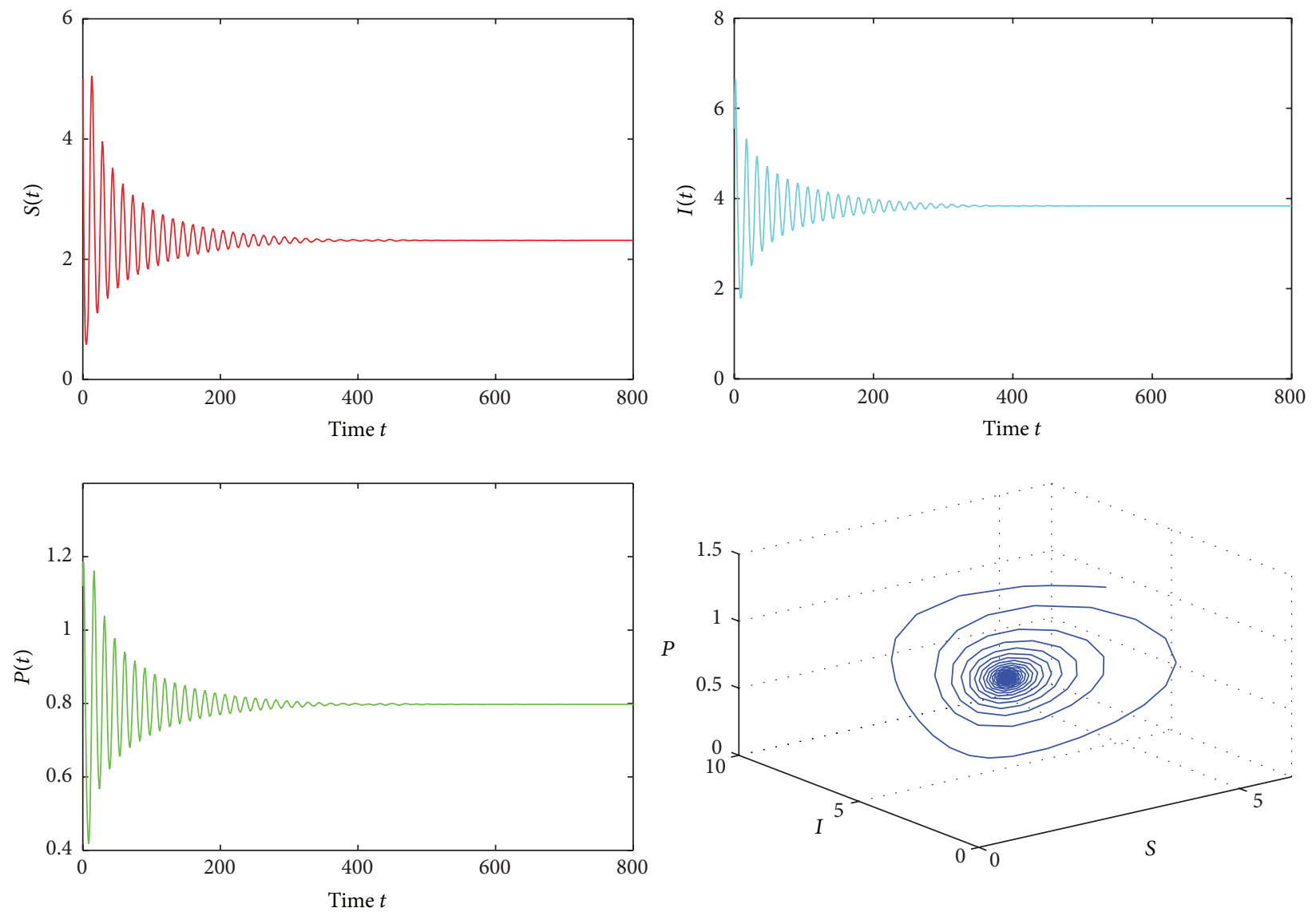

Figure 5: $E^{*}$ is asymptotically stable when $\tau=0.25<0.3652=\tau_{0}$.

$$
\begin{aligned}
& g_{11}=\tau_{20}^{*} \bar{D}\left[2 a_{21}+a_{22}\left(\bar{q}^{(2)}\left(-\frac{\tau_{10}^{*}}{\tau_{20}^{*}}\right)+q^{(2)}\left(-\frac{\tau_{10}^{*}}{\tau_{20}^{*}}\right)\right)\right. \\
& +\bar{q}_{3}^{*}\left(c_{21}\left(\bar{q}^{(3)}(0)\right)^{2}+c_{22} \bar{q}^{(1)}(-1) \bar{q}^{(3)}(0)\right. \\
& +a_{23}\left(q^{(3)}(0)+\bar{q}^{(3)}(0)\right) \\
& \left.\left.+c_{23}\left(\bar{q}^{(1)}(-1)\right)^{2}\right)\right] \\
& +\bar{q}_{2}^{*}\left(2 b_{21}+b_{22}\left(\bar{q}^{(2)}\left(-\frac{\tau_{10}^{*}}{\tau_{20}^{*}}\right)\right.\right. \\
& g_{21}=2 \tau_{20}^{*} \bar{D}\left[a_{21}\left(2 W_{11}^{(1)}(0)+W_{20}^{(1)}(0)\right)\right. \\
& \left.\left.+q^{(2)}\left(-\frac{\tau_{10}^{*}}{\tau_{20}^{*}}\right)\right)\right) \\
& +a_{22}\left(W_{11}^{(1)}(0) q^{(2)}\left(-\frac{\tau_{10}^{*}}{\tau_{20}^{*}}\right)+\frac{1}{2} W_{20}^{(1)}(0) \bar{q}^{(2)}\right. \\
& +\bar{q}_{3}^{*}\left(2 c_{21} q^{(3)}(0) \bar{q}^{(3)}(0)\right. \\
& +c_{22}\left(q^{(3)}(0) \bar{q}^{(1)}(-1)+\bar{q}^{(3)}(0) q^{(1)}(-1)\right) \\
& \times\left(-\frac{\tau_{10}^{*}}{\tau_{20}^{*}}\right)+W_{11}^{(2)}\left(-\frac{\tau_{10}^{*}}{\tau_{20}^{*}}\right) \\
& \left.+\frac{1}{2} W_{20}^{(2)}\left(-\frac{\tau_{10}^{*}}{\tau_{20}^{*}}\right)\right) \\
& \left.\left.+2 c_{23} q^{(1)}(-1) \bar{q}^{(1)}(-1)\right)\right] \\
& g_{02}=2 \tau_{20}^{*} \bar{D}\left[a_{21}+a_{22} \bar{q}^{(2)}\left(-\frac{\tau_{10}^{*}}{\tau_{20}^{*}}\right)+a_{23} \bar{q}^{(3)}(0)\right. \\
& +a_{23}\left(W_{11}^{(1)}(0) q^{(3)}(0)+\frac{1}{2} W_{20}^{(1)}(0) \bar{q}^{(3)}(0)\right. \\
& \left.+W_{11}^{(3)}(0)+\frac{1}{2} W_{20}^{(3)}(0)\right) \\
& +\bar{q}_{2}^{*}\left(b_{21}+b_{22} \bar{q}^{(2)}\left(-\frac{\tau_{10}^{*}}{\tau_{20}^{*}}\right)\right) \\
& +3 a_{31}+a_{32}\left(\bar{q}^{(2)}\left(-\frac{\tau_{10}^{*}}{\tau_{20}^{*}}\right)+2 q^{(2)}\left(-\frac{\tau_{10}^{*}}{\tau_{20}^{*}}\right)\right)
\end{aligned}
$$



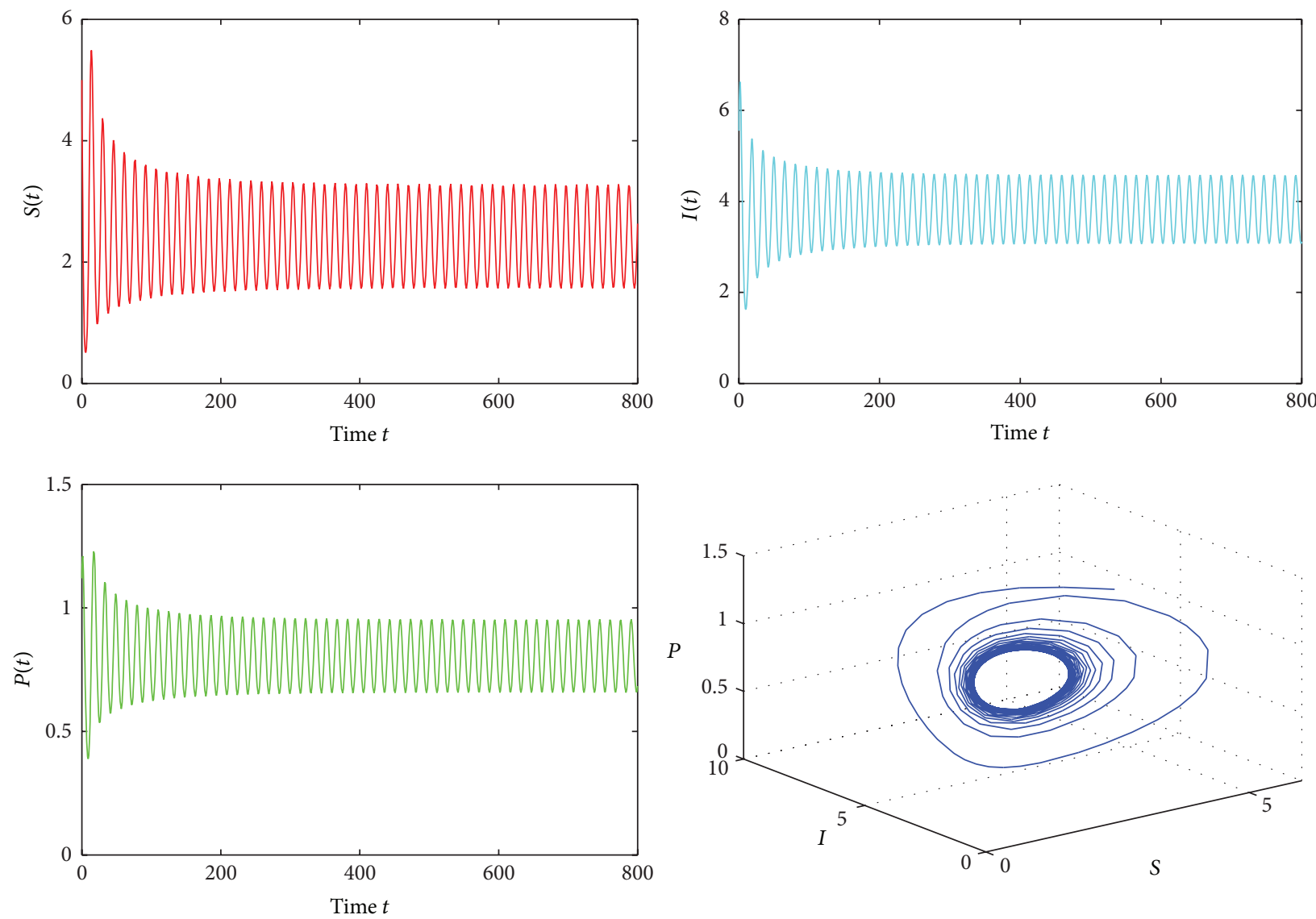

Figure 6: $E^{*}$ is unstable when $\tau=0.45>0.3652=\tau_{0}$.

$$
\begin{aligned}
& +a_{33}\left(\bar{q}^{(3)}(0)+2 q^{(3)}(0)\right) \\
& +\bar{q}_{2}^{*}\left(b_{21}\left(2 W_{11}^{(1)}(0)+W_{20}^{(1)}(0)\right)\right. \\
& +b_{22}\left(W_{11}^{(1)}(0) q^{(2)}\left(-\frac{\tau_{10}^{*}}{\tau_{20}^{*}}\right)+\frac{1}{2} W_{20}^{(1)}(0) \bar{q}^{(2)}\right. \\
& \times\left(-\frac{\tau_{10}^{*}}{\tau_{20}^{*}}\right)+W_{11}^{(2)}\left(-\frac{\tau_{10}^{*}}{\tau_{20}^{*}}\right) \\
& \left.+3 b_{31}+b_{32} W_{20}^{(2)}\left(-\frac{\tau_{10}^{*}}{\tau_{20}^{*}}\right)\right) \\
& \left.\times\left(\bar{q}^{(2)}\left(-\frac{\tau_{10}^{*}}{\tau_{20}^{*}}\right)+2 q^{(2)}\left(-\frac{\tau_{10}^{*}}{\tau_{20}^{*}}\right)\right)\right) \\
& +\bar{q}_{3}^{*}\left(c_{21}\left(2 W_{11}^{(3)}(0) q^{(3)}(0)+W_{20}^{(3)}(0) \bar{q}^{(3)}(0)\right)\right. \\
& +c_{22}\left(W_{11}^{(1)}(-1) q^{(3)}(0)+\frac{1}{2} W_{20}^{(1)}(-1)\right.
\end{aligned}
$$$$
\times \bar{q}^{(3)}(0)+W_{11}^{(3)}(0) q^{(1)}(-1)
$$$$
\left.+\frac{1}{2} W_{20}^{(3)}(0) \bar{q}^{(1)}(-1)\right)
$$$$
+c_{23}\left(2 W_{11}^{(1)}(-1)+W_{20}^{(1)}(-1)\right)
$$$$
+c_{31}\left(\left(q^{(1)}(-1)\right)^{2} \bar{q}^{(3)}(0)+2 q^{(1)}(-1)\right.
$$$$
\left.\times q^{(3)}(0) \bar{q}^{(1)}(-1)\right)
$$$$
\left.\left.+3 c_{32}\left(q^{(1)}(-1)\right)^{2} \bar{q}^{(1)}(-1)\right)\right]
$$

with

$$
\begin{gathered}
W_{20}(\theta)=\frac{i g_{20} q(0)}{\omega_{2 *} \tau_{20}^{*}} e^{i \omega_{2 *} \tau_{20}^{*} \theta}+\frac{i \bar{g}_{02} \bar{q}(0)}{3 \omega_{2 *} \tau_{20}^{*}} e^{-i \omega_{2 *} \tau_{20}^{*} \theta} \\
+E_{1} e^{2 i \omega_{2 *} \tau_{20}^{*} \theta}, \\
W_{11}(\theta)=-\frac{i g_{11} q(0)}{\omega_{2 *} \tau_{20}^{*}} e^{i \omega_{2 *} \tau_{20}^{*} \theta}+\frac{i \bar{g}_{11} \bar{q}(0)}{\omega_{2 *} \tau_{20}^{*}} e^{-i \omega_{2 *} \tau_{20}^{*} \theta}+E_{2},
\end{gathered}
$$



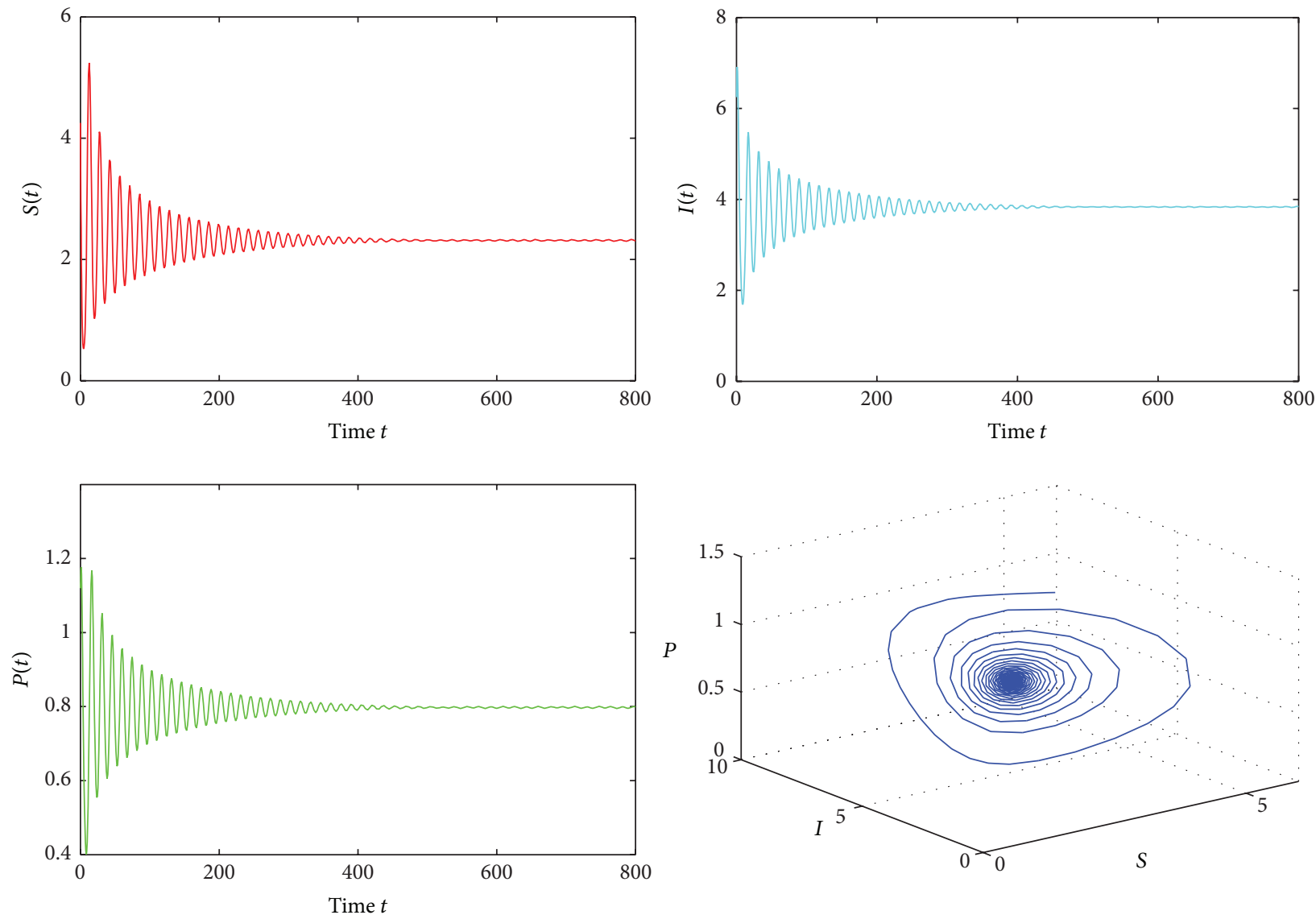

Figure $7: E^{*}$ is asymptotically stable when $\tau_{2}=0.75<1.0782=\tau_{20}^{*}$ and $\tau_{1}=0.15 \in\left(0, \tau_{10}\right)$.

where $E_{1}$ and $E_{2}$ can be computed as the following equations, respectively:

$$
\begin{gathered}
\left(\begin{array}{ccc}
2 i \omega_{2 *}-a_{1} & -b_{1} e^{-2 i \omega_{2 *} \tau_{20}^{*}} & -a_{2} \\
-a_{3} & 2 i \omega_{2 *}-a_{4}-b_{2} e^{-2 i \omega_{2 *} \tau_{20}^{*}} & 0 \\
-c_{1} e^{-2 i \omega_{2 *} \tau_{20}^{*}} & 0 & 2 i \omega_{2 *}-a_{5}
\end{array}\right) E_{1} \\
=2\left(\begin{array}{c}
E_{1}^{(1)} \\
E_{1}^{(2)} \\
E_{1}^{(3)}
\end{array}\right), \\
\left(\begin{array}{ccc}
a_{1} & b_{1} & a_{2} \\
a_{3} & a_{4}+b_{2} & 0 \\
c_{1} & 0 & a_{5}
\end{array}\right) E_{2}=-\left(\begin{array}{c}
E_{2}^{(1)} \\
E_{2}^{(2)} \\
E_{2}^{(3)}
\end{array}\right),
\end{gathered}
$$$$
E_{1}^{(3)}=c_{21}\left(q^{(3)}(0)\right)^{2}+c_{22} q^{(1)}(-1) q^{(3)}(0)+c_{23}\left(q^{(1)}(-1)\right)^{2} \text {, }
$$$$
E_{2}^{(1)}=2 a_{21}+a_{22}\left(\bar{q}^{(2)}\left(-\frac{\tau_{10}^{*}}{\tau_{20}^{*}}\right)+q^{(2)}\left(-\frac{\tau_{10}^{*}}{\tau_{20}^{*}}\right)\right)
$$$$
+a_{23}\left(q^{(3)}(0)+\bar{q}^{(3)}(0)\right) \text {, }
$$$$
E_{2}^{(2)}=2 b_{21}+b_{22}\left(\bar{q}^{(2)}\left(-\frac{\tau_{10}^{*}}{\tau_{20}^{*}}\right)+q^{(2)}\left(-\frac{\tau_{10}^{*}}{\tau_{20}^{*}}\right)\right) \text {, }
$$$$
E_{2}^{(3)}=2 c_{21} q^{(3)}(0) \bar{q}^{(3)}(0)
$$$$
+c_{22}\left(q^{(3)}(0) \bar{q}^{(1)}(-1)+\bar{q}^{(3)}(0) q^{(1)}(-1)\right)
$$$$
+2 c_{23} q^{(1)}(-1) \bar{q}^{(1)}(-1) \text {. }
$$

with

$$
\begin{aligned}
& E_{1}^{(1)}=a_{21}+a_{22} q^{(2)}\left(-\frac{\tau_{10}^{*}}{\tau_{20}^{*}}\right)+a_{23} q^{(3)}(0), \\
& E_{1}^{(2)}=b_{21}+b_{22} q^{(2)}\left(-\frac{\tau_{10}^{*}}{\tau_{20}^{*}}\right),
\end{aligned}
$$

Therefore, we can calculate the following values: 

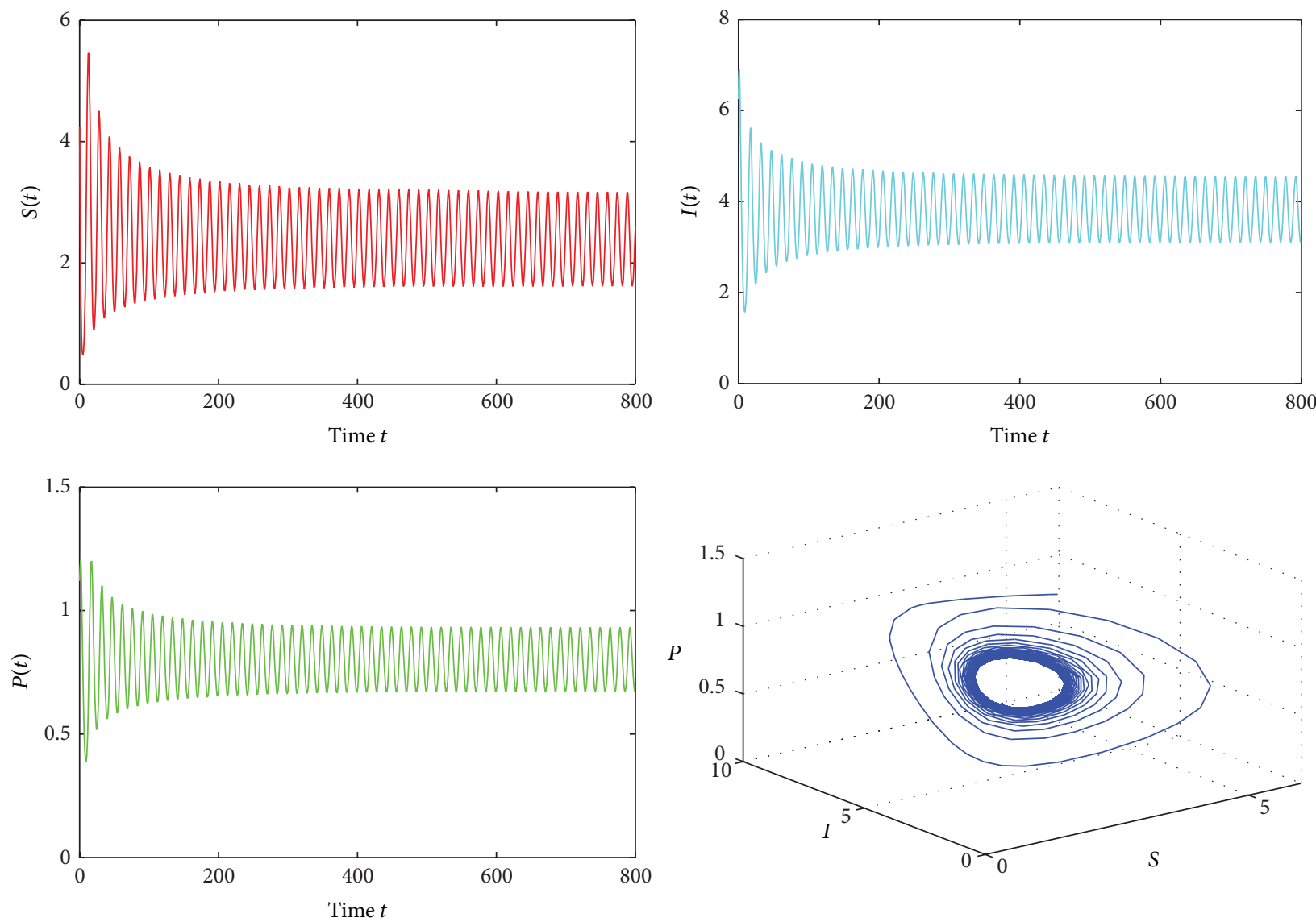

Figure 8: $E^{*}$ is unstable when $\tau_{2}=1.35>1.0782=\tau_{20}^{*}$ and $\tau_{1}=0.15 \in\left(0, \tau_{10}\right)$.

$$
\begin{gathered}
\beta_{2}=2 \operatorname{Re}\left\{C_{1}(0)\right\}, \\
T_{2}=-\frac{\operatorname{Im}\left\{C_{1}(0)\right\}+\mu_{2} \operatorname{Im}\left\{\lambda^{\prime}\left(\tau_{20}^{*}\right)\right\}}{\omega_{2 *} \tau_{20}^{*}} .
\end{gathered}
$$

Based on the discussion above, we can obtain the following results.

Theorem 6. For system (2),

(i) $\mu_{2}$ determines the direction of the Hopf bifurcation. If $\mu_{2}>0\left(\mu_{2}<0\right)$, then the Hopf bifurcation is supercritical (subcritical).

(ii) $\beta_{2}$ determines the stability of the bifurcating periodic solutions. If $\beta_{2}<0\left(\beta_{2}>0\right)$, then the bifurcating periodic solutions are stable (unstable).

(iii) $T_{2}$ determines the period of the bifurcating periodic solutions. If $T_{2}>0\left(T_{2}<0\right)$, then the period of the bifurcating periodic solutions increases (decreases).

\section{Numerical Example}

In this section, we give a numerical example to support the theoretical results in Sections 2 and 3. We use the same coefficients which are used by Jana and Kar in [6]. They are as follows: $a=5.1, b=3.995, m=0.8, r=1.1, K=8.8$, $\alpha=1.25, \beta=1.3, \gamma=0.39, \delta=0.29$, and $\varepsilon=0.15$. Thus, we get the following particular case of system (2):

$$
\begin{gathered}
\frac{d S(t)}{d t}=1.1 S\left(1-\frac{S}{8.8}\right)-\frac{1.25 S I\left(t-\tau_{1}\right)}{5.1+S}-\frac{1.3 S P}{3.995+S}, \\
\frac{d I(t)}{d t}=\frac{1.25 S I\left(t-\tau_{1}\right)}{5.1+S}-0.39 I, \\
\frac{d P(t)}{d t}=P\left[\frac{1.04 S\left(t-\tau_{2}\right)}{3.995+S\left(t-\tau_{2}\right)}-0.29 P-0.15\right],
\end{gathered}
$$

which has a positive equilibrium $E^{*}(2.3128,3.8339,0.7977)$.

For $\tau_{1}>0, \tau_{2}=0$. We get $\omega_{10}=0.4149<0$, $\tau_{10}=0.5148$. Further, we have $f_{1}^{\prime}\left(v_{1 *}\right)=0.1022>0$. Thus, conditions $\left(H_{21}\right)$ and $\left(H_{22}\right)$ hold. From Theorem 2 , the positive equilibrium $E^{*}$ is asymptotically stable when $\tau_{1}<\tau_{10}$ as illustrated by Figure 1 . When $\tau_{1}$ passes through the critical value $\tau_{10}$, the positive equilibrium $E^{*}$ loses its stability and a Hopf bifurcation occurs and a family of periodic solutions bifurcate from the positive equilibrium $E^{*}$, which can be shown as in Figure 2. Similarly, we have $\omega_{20}=0.6010, \tau_{20}=$ 1.8829 for $\tau_{1}=0, \tau_{2}>0$. The corresponding waveforms and the phase plots are shown in Figures 3 and 4. 
For $\tau_{1}=\tau_{2}=\tau>0$, we can obtain $\omega_{0}=2.0030$ and then we get $\tau_{0}=0.3652$. From Theorem 4 , we know that when $\tau$ increases from zero to the critical value $\tau_{0}$ the positive equilibrium $E^{*}$ is asymptotically stable; then it will lose its stability and a Hopf bifurcation occurs once $\tau>\tau_{0}$. These properties can be shown as in Figures 5 and 6.

For $\tau_{2}>0$ and $\tau_{1}=0.15 \in\left(0, \tau_{10}\right)$, we can obtain $\omega_{2 *}=2.1105, \tau_{20}^{*}=1.0782$. By Theorem 5 , the positive equilibrium $E^{*}$ is asymptotically stable when $\tau_{2} \in\left[0, \tau_{20}^{*}\right)$ and $E^{*}$ is unstable when $\tau_{2}>\tau_{20}^{*}$ and a Hopf bifurcation occurs, which can be illustrated by Figures 7 and 8 . In addition, by complex computations, we obtain $C_{1}(0)=-2.2071+1.6159 i$, and further we have $\mu_{2}=6.8522>0, \beta_{2}=-4.4142<0$, $T_{2}=-3.7367<0$. By Theorem 6 , we know that the Hopf bifurcation with respect to $\tau_{2}$ with $\tau_{1}=0.15 \in\left(0, \tau_{10}\right)$ is supercritical; the bifurcating periodic solutions are stable and decrease. From the viewpoint of ecology, if the periodic solutions bifurcating from the Hopf bifurcation are stable, the species in a prey-predator system may coexist in an oscillatory mode. Therefore, we can conclude that the three species in system (75) can coexist in an oscillatory mode, since the bifurcating periodic solutions are stable.

\section{Conclusion}

In this present paper a prey-predator system with disease in the prey and two delays is considered. Based on the system proposed in [6], we further incorporate the time delay due to the gestation of the predator. The main purpose of this paper is to investigate the effects of the two delays on the system. We have shown that the two delays play a complicated role in the system. By choosing the possible combinations of the two delays as bifurcation parameters, sufficient conditions for local stability and existence of local Hopf bifurcation are obtained. When the time delay is below the corresponding critical value, we get that the system is local stable. Otherwise, a local Hopf bifurcation occurs at the positive equilibrium. We also find that the delay due to the susceptible prey becoming the infected prey is more marked compared with the delay due to the gestation of the predator, because the critical value of $\tau_{1}$ is much smaller than that of $\tau_{2}$ when we only consider one of the two delays, which can be seen from the numerical simulations. Further, the properties of the bifurcated periodic solutions such as the direction and the stability are determined. And a numerical example is also given to support the theoretical results. From the numerical simulations we can see that the species in the system considered in this paper can coexist under some certain conditions.

\section{Conflict of Interests}

The author declares that there is no conflict of interests regarding the publication of this paper.

\section{Acknowledgments}

The author is grateful to the referees and the editor for their valuable comments and suggestions on the paper. This work was supported by the Natural Science Foundation of the Higher Education Institutions of Anhui Province (KJ2013A003, KJ2013B137).

\section{References}

[1] Y. Xiao and L. Chen, "Analysis of a three species eco-epidemiological model," Journal of Mathematical Analysis and Applications, vol. 258, no. 2, pp. 733-754, 2001.

[2] S. Sun and C. Yuan, "On the analysis of predator-prey model with epidemic in the predator," Journal of Biomathematics, vol. 21, no. 1, pp. 97-104, 2006.

[3] J.-F. Zhang, W.-T. Li, and X.-P. Yan, "Hopf bifurcation and stability of periodic solutions in a delayed eco-epidemiological system," Applied Mathematics and Computation, vol. 198, no. 2, pp. 865-876, 2008.

[4] S. Chakraborty, S. Pal, and N. Bairagi, "Dynamics of a ratiodependent eco-epidemiological system with prey harvesting," Nonlinear Analysis: Real World Applications, vol. 11, no. 3, pp. 1862-1877, 2010.

[5] G.-P. Hu and X.-L. Li, "Stability and Hopf bifurcation for a delayed predator-prey model with disease in the prey," Chaos, Solitons \& Fractals, vol. 45, no. 3, pp. 229-237, 2012.

[6] S. Jana and T. K. Kar, "Modeling and analysis of a prey-predator system with disease in the prey," Chaos, Solitons \& Fractals, no. 47, pp. 42-53, 2013.

[7] T. Saha and C. Chakrabarti, "Dynamical analysis of a delayed ratio-dependent Holling-Tanner predator-prey model," Journal of Mathematical Analysis and Applications, vol. 358, no. 2, pp. 389-402, 2009.

[8] S. Guo and W. Jiang, "Hopf bifurcation analysis on general Gause-type predator-prey models with delay," Abstract and Applied Analysis, vol. 2012, Article ID 363051, 17 pages, 2012.

[9] S. Jana, M. Chakraborty, K. Chakraborty, and T. K. Kar, "Global stability and bifurcation of time delayed prey-predator system incorporating prey refuge," Mathematics and Computers in Simulation, vol. 85, pp. 57-77, 2012.

[10] X. Liu and M. Han, "Chaos and Hopf bifurcation analysis for a two species predatorprey system with prey refuge and diffusion," Nonlinear Analysis: Real World Applications, vol. 12, no. 2, pp. 1047-1061, 2011.

[11] M. Ferrara, L. Guerrini, and C. Bianca, "The Cai model with time delay: existence of periodic solutions and asymptotic analysis," Applied Mathematics \& Information Sciences, vol. 7, no. 1, pp. 21-27, 2013.

[12] S. Gakkhar and A. Singh, "Complex dynamics in a prey predator system with multiple delays," Communications in Nonlinear Science and Numerical Simulation, vol. 17, no. 2, pp. 914-929, 2012.

[13] Z. Z. Zhang, H. Z. Yang, and J. Liu, "Stability and Hopf bifurcation in a modified Holling-Tanner predator-prey system with multiple delays," Abstract and Applied Analysis, vol. 2012, Article ID 236484, 19 pages, 2012.

[14] X.-Y. Meng, H.-F. Huo, and H. Xiang, "Hopf bifurcation in a three-species system with delays," Journal of Applied Mathematics and Computing, vol. 35, no. 1-2, pp. 635-661, 2011.

[15] J. Liu, C. W. Sun, and Y. M. Li, "Stability and Hopf bifurcation analysis for a Gause-type predator-prey system with multiple delays," Abstract and Applied Analysis, vol. 2013, Article ID 795358, 12 pages, 2013.

[16] T. K. Kar and A. Ghorai, "Dynamic behaviour of a delayed predator-prey model with harvesting," Applied Mathematics and Computation, vol. 217, no. 22, pp. 9085-9104, 2011. 
[17] Y. L. Song, M. Han, and J. Wei, "Stability and Hopf bifurcation analysis on a simplified BAM neural network with delays," Physica D, vol. 200, no. 3-4, pp. 185-204, 2005.

[18] B. D. Hassard, N. D. Kazarinoff, and Y. H. Wan, Theory and Applications of Hopf Bifurcation, Cambridge University Press, Cambridge, UK, 1981. 


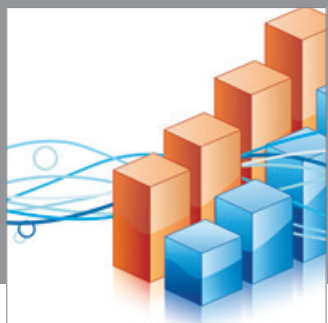

Advances in

Operations Research

mansans

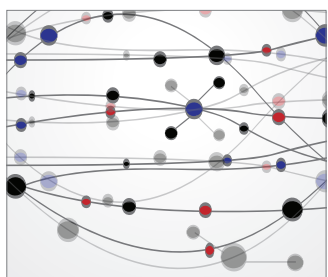

The Scientific World Journal
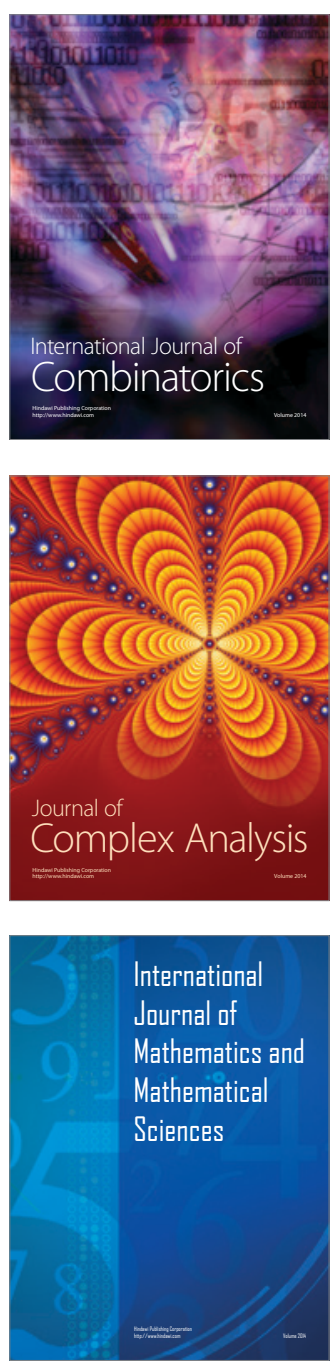
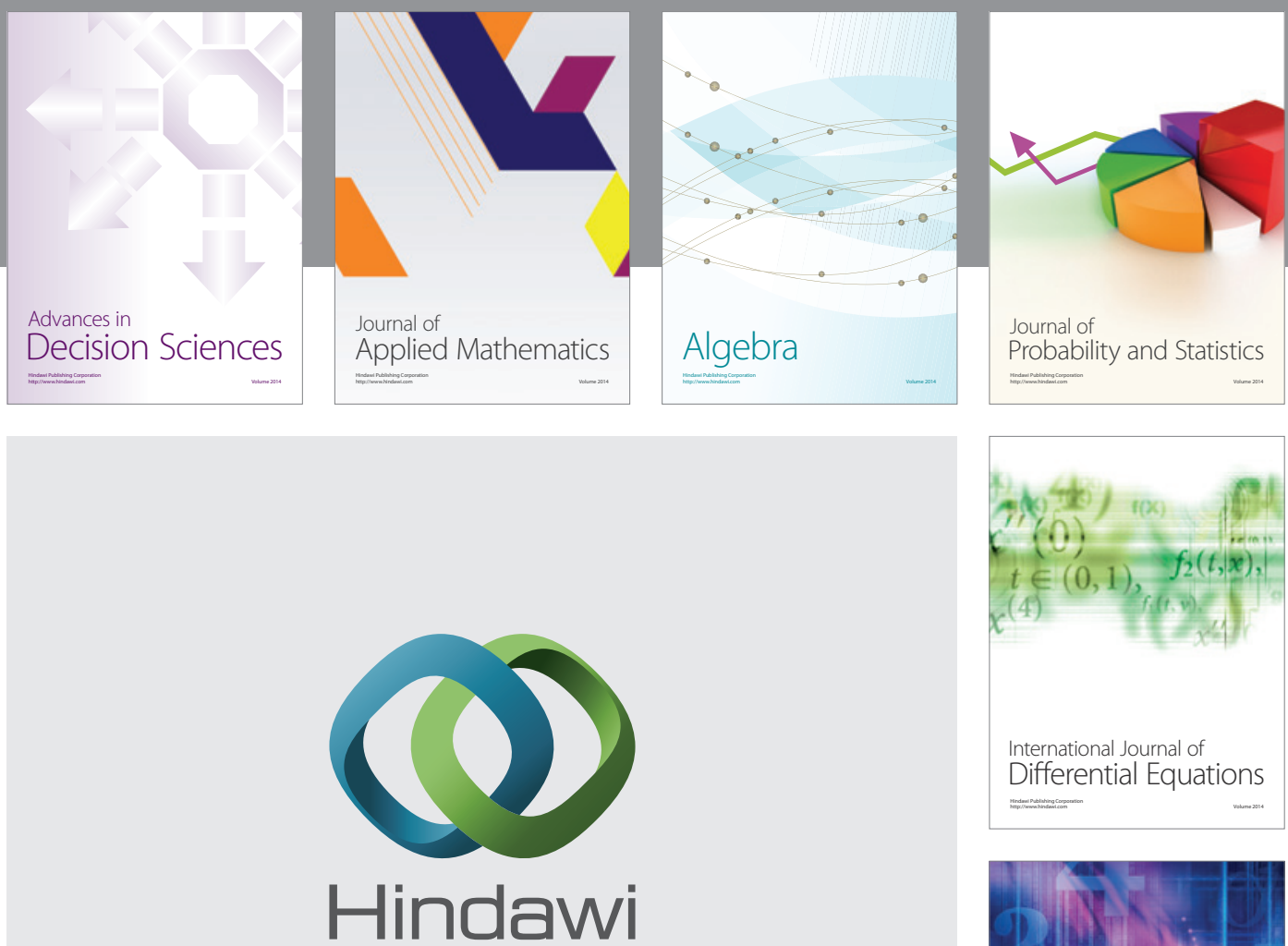

Submit your manuscripts at http://www.hindawi.com
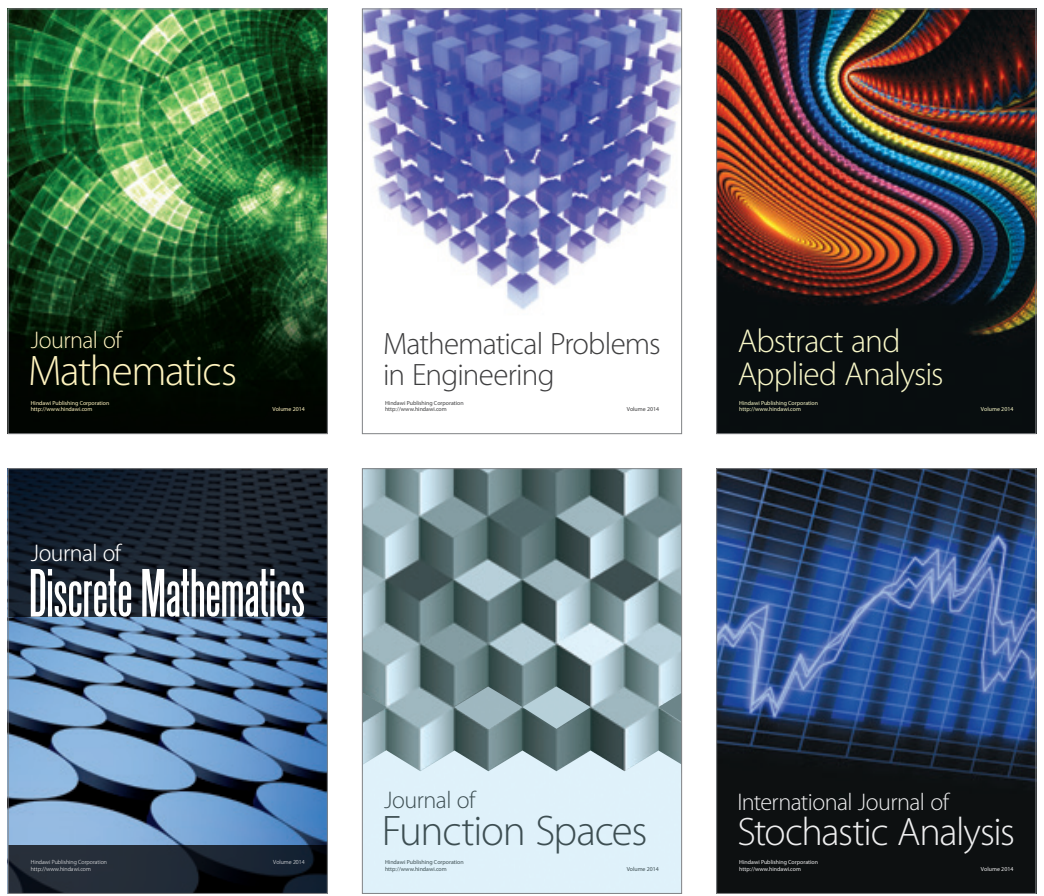

Journal of

Function Spaces

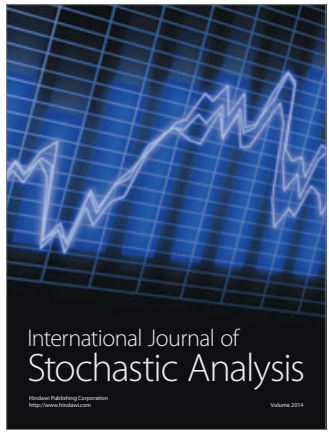

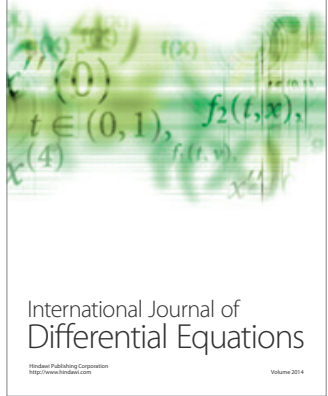
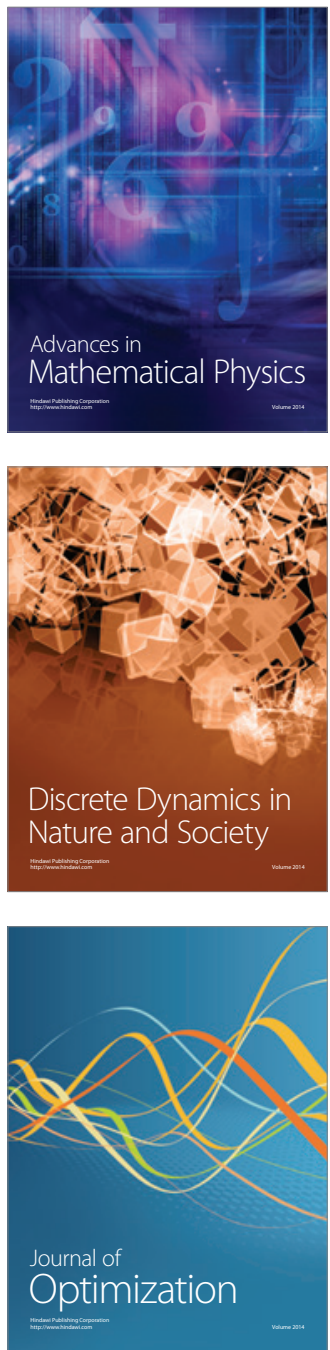